NISTIR 7632

\title{
Bochner Subordination, Logarithmic Diffusion Equations, and Blind Deconvolution of Hubble Space Telescope Imagery and Other Scientific Data
}

Alfred S. Carasso

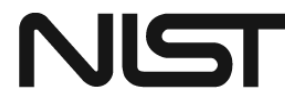


NISTIR 7632

\title{
Bochner Subordination, Logarithmic Diffusion Equations, and Blind Deconvolution of Hubble Space Telescope Imagery and Other Scientific Data
}

\author{
Alfred S. Carasso \\ Mathematical and Computational Sciences Division \\ Information Technology Laboratory
}

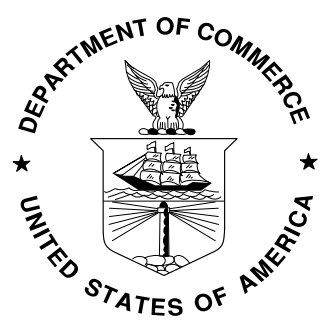

U.S. Department of Commerce Gary Locke, Secretary 


\title{
BOCHNER SUBORDINATION, LOGARITHMIC DIFFUSION EQUATIONS, AND BLIND DECONVOLUTION OF HUBBLE SPACE TELESCOPE IMAGERY AND OTHER SCIENTIFIC DATA
}

\author{
ALFRED S. CARASSO*
}

\begin{abstract}
Generalized Linnik processes and associated logarithmic diffusion equations can be constructed by appropriate Bochner randomization of the time variable in Brownian motion and the related heat conduction equation. Remarkably, over a large but finite frequency range, generalized Linnik characteristic functions can exhibit almost Gaussian behavior near the origin, while behaving like low exponent isotropic Lévy stable laws away from the origin. Such behavior matches Fourier domain behavior in a large class of real blurred images of considerable scientific interest, including Hubble space telescope imagery and scanning electron micrographs. This paper develops a powerful blind deconvolution procedure based on postulating system optical transfer functions (otf) in the form of generalized Linnik characteristic functions. The system otf and 'true' sharp image are then reconstructed by solving a related logarithmic diffusion equation backwards in time, using the blurred image as data at time $t=1$. The present methodology significantly improves upon previous work based on system otfs in the form of Lévy stable characteristic functions. Such improvement is validated by the substantially smaller image Lipschitz exponents that ensue, confirming increased fine structure recovery. These results resolve the unexplained appearance of exceptionally low Lévy stable exponents in previous work on the same class of images. The paper is illustrated with striking enhancements of gray scale and colored images.
\end{abstract}

Key words. image deblurring; blind deconvolution; Bochner subordination; generalized Linnik laws; low exponent Lévy stable laws; fractional and logarithmic diffusion equations; Lipschitz exponents; Hubble space telescope; scanning electron microscope; Whirlpool galaxy; Starburst galaxy.

AMS subject classifications. 35R25, 35B60, 60E07, 68U10.

1. Introduction. This paper deals with blind deconvolution of a class of real blurred images of considerable scientific interest, as opposed to synthetically degraded phantoms. A preselected class of trial optical transfer functions (otfs), not previously known in image analysis, is shown to produce striking enhancements of Hubble space telescope images and other astronomical data, as well as useful sharpening of scanning electron micrographs of interest in Nanotechnology. Such improvements may not be apparent in the reduced size images in the printed issue of this journal. However, significant enhancement becomes evident when the on-line version of this paper is viewed at full size on a modern high resolution device, such as a wide screen, active matrix, liquid crystal display (LCD) monitor. As noted below, this degree of fine structure reconstruction is difficult to achieve with existing techniques.

Blind deconvolution seeks to deblur an image without knowing the cause of the blur. The methodology used here is based on identifying a plausible blurring otf from within a restricted class of candidate blurs. The choice of trial otfs is governed by the Fourier domain behavior in the blurred imagery under consideration. Previous work on the above class of problems [10], [11], was based on candidate otfs in the form of isotropic Lévy stable characteristic functions [18], [27], [31], and the use of timereversed diffusion equations involving fractional powers of the negative Laplacian. An important observation in [7], [10], [11], is that the successful deblurring otfs are characterized by low Lévy exponents, with typical values less than 0.5 , while Gaussian otfs have exponent 2. Such low values are quite exceptional in applications where Lévy stable laws appear. The physical origin, if any, of such exponent values is not known.

\footnotetext{
* Computing and Mathematical Sciences Laboratory, National Institute of Standards and Technology, Gaithersburg, MD 20899. (alfred.carasso@nist.gov).
} 
The present method is based on otfs in the form of generalized Linnik characteristic functions [16], [26], [32], and the use of time-reversed diffusion equations involving the logarithm of the negative Laplacian plus the identity. We show that this results in higher quality reconstructions than previously obtained. This improvement is validated by the substantially smaller image Lipschitz exponents that ensue, confirming increased fine structure recovery [8], [13]. Moreover, the high frequency behavior in the successful Linnik deblurring otfs resolves the unexplained appearance of low Lévy exponents in [10] and [11].

While Linnik otfs have distinctly different behavior near the origin than do Lévy stable otfs, there is a close connection between these two classes of objects. This connection centers on the notion of Bochner subordination of stochastic processes [5], [6], [18], [32], whereby a given Markov process $\mathbf{X}(t), t>0$, is observed in new stochastic 'operational time' $\mathbf{S}(t)$, rather than in standard clock time $t$, resulting in the process $\mathbf{X}(\mathbf{S}(t))$. This concept has found fruitful application in several branches of science, engineering, and finance [1], [15], [19], [20], [35]. In that context, generalized Linnik characteristic functions describe the family of processes obtained when isotropic Lévy stable motions are observed in the randomized operational time $\boldsymbol{\Gamma}(t)$, where for each $t>0, \boldsymbol{\Gamma}(t)$ obeys a Gamma distribution on $u \geq 0$. The important special case of Brownian motion observed in stochastic time $\boldsymbol{\Gamma}(t)$ is called the Variance Gamma Process [28], [29]. This has been used successfully in option pricing.

Bochner subordination also plays a role in operator semigroup theory and evolutionary partial differential equations. By combining subordination with the HilleYosida theorem, a functional calculus for semigroup generators is developed in [30], whereby entirely new semigroups can be created by randomizing the time variable. In [12], it is shown that the subordinator $\boldsymbol{\Gamma}(t)$ discussed above is one of a large family of stochastic time changes with the remarkable property of converting all $C_{0}$ semigroups on a given complex Banach space $B$, into holomorphic semigroups on $B$.

In this paper, the use of generalized Linnik densities as trial blurring kernels is primarily motivated by their mathematical properties. The semigroup property of infinite divisibility [18], [27], [32], the Fourier domain Gaussian-like behavior near the origin, together with the monotone convex high frequency behavior which imitates the blurred image data, all play essential roles. We do not claim an actual physical basis for Linnik otfs, and the significance of this paper ultimately rests on the quality of the reconstructions. However, our results strongly suggest a possible physical basis for such Linnik otfs, mediated by subordination as in previously mentioned applications. While Gaussian otfs, synonymous with Brownian motion, are a standard first choice in image reconstruction, image acquisition involves the interaction of several interfacing optical and electronic devices, each producing a small distortion of the input signal. Additional aberrations may result from the scattering properties of the medium through which radiation propagates. A significant empirical discovery [21], [22], [23], is that a large variety of electro-optical imaging devices have otfs given by Lévy stable characteristic functions. Very recently [3], an optical material was created with a specific kind of inhomogeneity, in which the scattering of light waves results in Lévy flights rather than Brownian motion. A prescribed Lévy exponent for this scattering process can be engineered by proper synthesis of the inhomogeneity. As is well-known, Lévy stable motions are related to Brownian motion through Bochner subordination. A second subordination, involving the $\boldsymbol{\Gamma}(t)$ process, would result in generalized Linnik otfs. Conceivably, Linnik otfs may play an unsuspected role in many imaging situations. 
The blind deconvolution procedure presented here is fundamentally different from such variational blind formulations as [14] and [24]. These methods aim to solve the blind deconvolution problem in full generality, by minimizing an appropriate cost functional. Such techniques do not appear useful for the present class of problems. The method in [14] is primarily intended for 'blocky' images, and point spread functions with edges. Other promising approaches such as [2], exploit prior knowledge of the edge map in the unknown image. Astronomical images are typically diffuse and partly amorphous, and are very far from being of bounded variation. Use of [14] tends to eliminate individual star clusters and other localized bright areas, which are interpreted as unwanted noise. The method in [24] requires a prior guess for the system otf. However, even with a good guess, this method often returns a questionable sharp image associated with a new, but physically impossible otf [9]. If and when comprehensive blind deconvolution methods become available, the methodology presented here is likely to remain useful. It can provide independent reconstructions for comparison, and it can provide valuable initial guesses that might be refined by more elaborate procedures.

2. Imagery with monotone convex Fourier decay. All images in this paper are 8-bit images, with pixel values scaled from 0 to 255 . Given an image $g(x, y)$, we define its Fourier transform by

$$
\hat{g}(\xi, \eta) \equiv \int_{R^{2}} g(x, y) e^{-2 \pi i(\xi x+\eta y)} d x d y,
$$

and we denote by $\|g\|_{r}$ the $L^{r}\left(R^{2}\right)$ norm of $g(x, y)$. Typically, $r=1$ or 2 . We also use the 'total variation' seminorm

$$
\|g\|_{T V} \equiv\|\nabla g\|_{1} .
$$

In general, a real blurred image $g(x, y)$ contains noise $n(x, y)$ which may be multiplicative,

$$
g(x, y)=g_{e}(x, y)+n(x, y), \quad\|n\|_{r} \ll\left\|g_{e}\right\|_{r}, \quad r=1,2,
$$

where $g_{e}(x, y)$ denotes the exact blurred image that would have been recorded in the absence of any errors or noise. Neither $g_{e}(x, y)$ nor $n(x, y)$ are known, only their sum $g(x, y)$.

The example in Figure 1 is illustrative of a large class of images of galaxies and other astronomical objects. An original $512 \times 512$ pixels Pleiades (M45) image $g(x, y)$, is shown on the left hand side of Figure 1. Of particular interest is the plot of $\log |\hat{g}(\xi, 0)|$ vs $|\xi|$, shown as the dashed curve immediately below. While that trace is locally highly oscilllatory, it is globally monotone decreasing and convex. The solid curve $p(\xi)$ captures the gross behavior in $\log |\hat{g}(\xi, 0)|$ away from the origin. The deblurred image on the right hand side is a good representation of a sharp Pleiades image $f(x, y)$, and we shall consider $f(x, y)$ to be the 'true' Pleiades image for the purpose of this discussion. We note that $\log |\hat{f}(\xi, 0)|$ exhibits a very similar globally monotone convex trace, whose gross behavior is captured by the solid curve $q(\xi)$. The curve $p$ decays faster than the curve $q$. Both $p$ and $q$ were chosen so as to have a maximum value of 0 , attained at $\xi=0$. In addition, as shown in the bottom drawing in Figure 1, the curves $\log |\hat{g}(\xi, 0)|$ and $\log |\hat{f}(\xi, 0)|$ are such that away from the origin, the ratio of these two quantities remains fairly constant, fluctuating around 


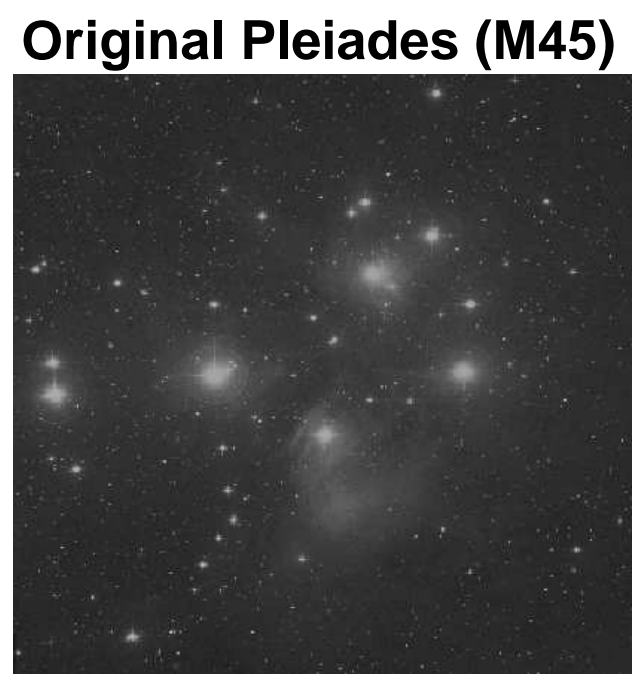

True Pleiades?

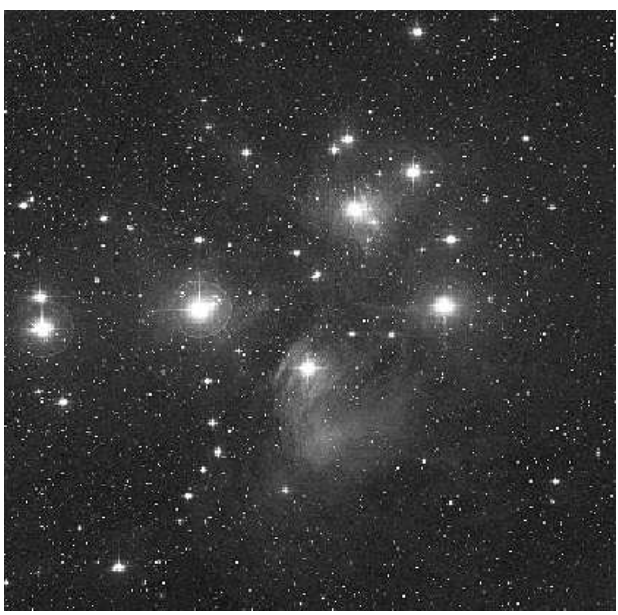

\section{Behavior in Fourier transform domain}
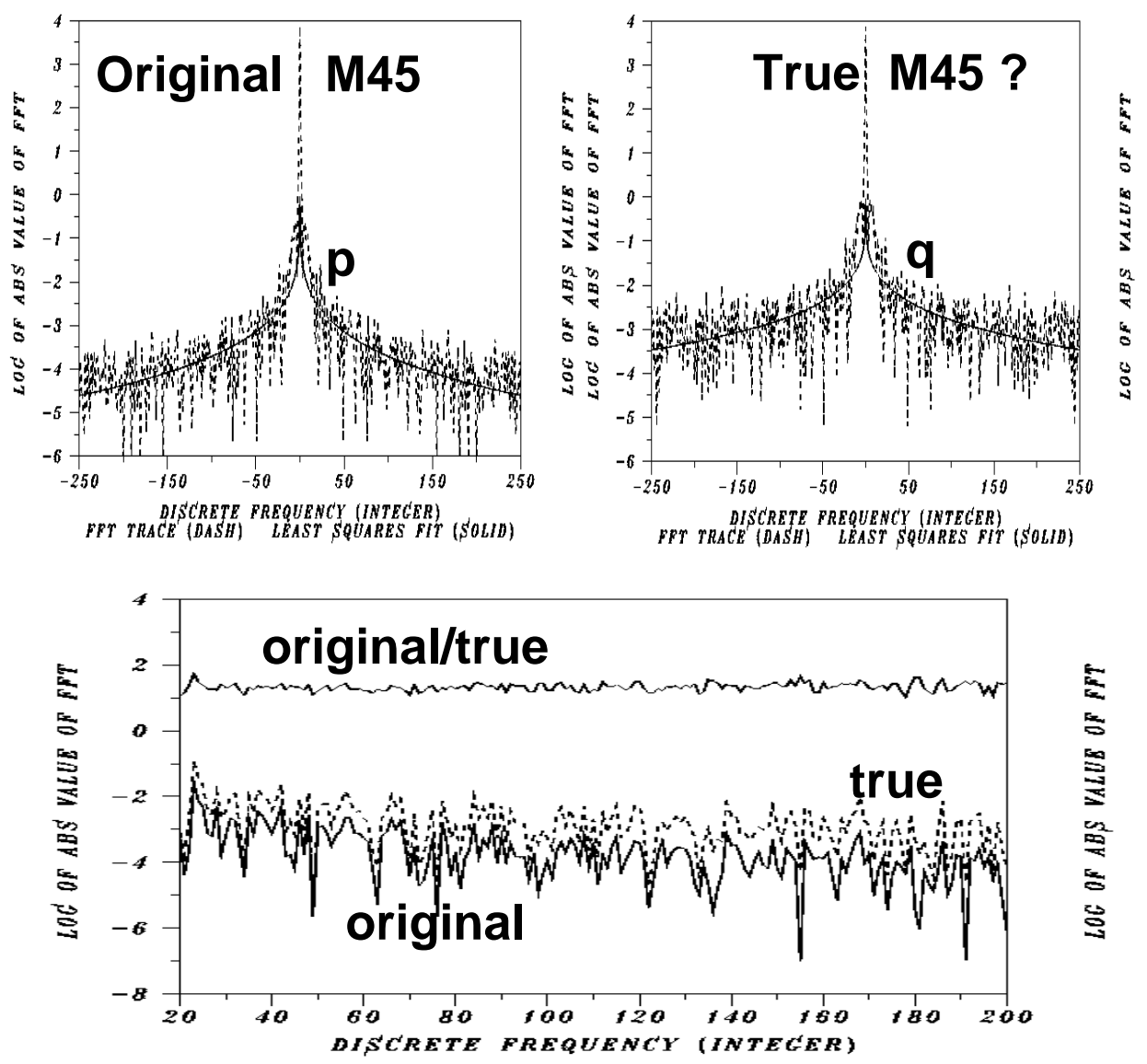

FIG. 1. Above Pleiades Fourier transform behavior is representative of a large class of images. Away from the origin, blurred image data $\log |\hat{g}(\xi, 0)|$ on left, and 'true' image data $\log |\hat{f}(\xi, 0)|$ on right, exhibit similar highly oscillatory globally convex monotone behavior, but left hand data decays faster. Moreover, the ratio $\log |\hat{g}(\xi, 0)| / \log |\hat{f}(\xi, 0)|$ remains fairly constant, fluctuating around some mean value larger than unity. 
some mean value larger than 1 . Accordingly,

$$
\begin{aligned}
& \log |\hat{g}(\xi, 0)| / \log |\hat{f}(\xi, 0)| \approx \text { Constant }>1, \quad|\xi| \gg 1, \\
& p(\xi) / q(\xi) \approx \text { Constant }>1, \quad|\xi| \gg 1 .
\end{aligned}
$$

A comparison of the left and right hand sides in Figure 1 invites the following, possibly naive, interpretation. Assume that the original image $g(x, y)$ on the left is a blurred version of the true image $f(x, y)$ on the right, and results from the convolution of $f(x, y)$ with an isotropic, shift invariant, point spread function $h(x, y)$. Note that $h(x, y)$ is a $2 \mathrm{D}$ probability density function since it is non-negative and integrates to unity. The Fourier transform of $h(x, y)$ is the optical transfer function $\hat{h}(\xi, \eta)$. That object is necessarily a 2D characteristic function and must obey Bochner's theorem on positive definite functions [4], [17]. Since $h(x, y)$ is isotropic, so is $\hat{h}(\xi, \eta)$, and we write

$$
\hat{h}(\xi, \eta)=\hat{h}(\rho), \quad \rho=\sqrt{\xi^{2}+\eta^{2}} .
$$

Assume further that the characteristic function $\hat{h}(\rho)$ is positive and monotone decreasing. This is the case for a large class of isotropic otfs, based on Schoenberg's theorem on completely monotone functions [17], [33]. With $\otimes$ denoting convolution in $L^{1}\left(R^{2}\right)$, we have

$$
\begin{aligned}
& g_{e}(x, y)+n(x, y) \equiv g(x, y)=h(x, y) \otimes f(x, y), \\
& \log |\hat{g}(\xi, \eta)|=\log \hat{h}(\rho)+\log |\hat{f}(\xi, \eta)|, \\
& \log |\hat{g}(\xi, 0)| \sim p(\xi), \quad \log |\hat{f}(\xi, 0)| \sim q(\xi), \quad|\xi| \gg 1, \\
& \log \hat{h}(\rho) \sim p(\rho)-q(\rho), \quad \rho \gg 1 .
\end{aligned}
$$

Thus, under the preceding assumptions, behavior in $\hat{h}(\rho)$ away from the origin can be inferred from that in $p-q$. In practice, given an original image $g(x, y)$ with Fourier domain behavior similar to that on the left in Figure 1, we may find $p(\xi)$ but not $q(\xi)$. However, for a large class of blurred images $g(x, y)$, the a-priori assumption that the corresponding true image $f(x, y)$ has Fourier behavior similar to that on the right of Figure 1, and obeys (4), is found to lead to useful reconstructions. Accordingly, using (4) and (6), we postulate that with some unknown positive constant $c<1$

$$
\log \hat{h}(\rho) \sim(1-c) p(\rho), \quad \rho \gg 1 .
$$

This paper seeks to identify the system otf by a least squares fit to the data $\log |\hat{g}(\xi, 0)|$ with a suitable monotone decreasing function $p(\xi)$ as above, with $p(0)=0$. That function must be such that for any constant $b$ with $0<b<1$, the function $b p(\rho)$ is the logarithm of a characteristic function. This is the case if and only if $\exp \{p(\rho)\}$ is an infinitely divisible characteristic function [18], [27].

3. Infinitely divisible otfs and subordinated diffusion equations. In the deconvolution problem $h(x, y) \otimes f(x, y)=g(x, y)$ to be analyzed below, the targeted class of images directs attention to the following three types of infinitely divisible 
isotropic otf

$$
\begin{aligned}
& \hat{h}(\rho)=\exp \left(-\sigma \rho^{2}\right), \quad \sigma>0, \quad \rho=\sqrt{\xi^{2}+\eta^{2}}, \quad \text { Gaussian } \\
& \hat{h}(\rho)=\exp \left(-\sigma \rho^{\alpha}\right), \quad \sigma>0, \quad 0<\alpha \leq 2, \quad \text { Lévy stable } \\
& \hat{h}(\rho)=\left(1+\sigma \rho^{\alpha}\right)^{-\lambda}, \quad \sigma, \lambda>0, \quad 0<\alpha \leq 2 . \quad \text { Generalized Linnik }
\end{aligned}
$$

Each of these three types is associated with a diffusion equation. The Gaussian case, representing Brownian motion, corresponds to the heat equation as is wellknown. The other two types involve stochastic processes and corresponding diffusion equations that result from randomizing the time variable in Brownian motion. We show this by introducing the notion of subordinated semigroup [12], [30], [32].

Consider a family of functions $\left\{p_{t}(u)\right\}$ indexed by $t \geq 0$ and defined on $u \geq 0$, and such that for each fixed $t>0, p_{t}(u)$ is a probability density function on $u \geq 0$. Assume that $p_{t}(u) * * p_{s}(u)=p_{t+s}(u)$, where $* *$ denotes convolution on $u \geq 0$, and that $p_{t}(u) \rightarrow \delta(u)$ as $t \downarrow 0$. For fixed $t>0$, the Laplace transform of $p_{t}(u)$ is defined by

$$
\mathcal{L}\left(p_{t}\right)=\int_{0}^{\infty} p_{t}(u) e^{-u z} d u, \quad \text { Re } z>0
$$

Definition 1. A Bochner subordinator is a family $\left\{p_{t}(u)\right\}$ as defined above, such that $\mathcal{L}\left(p_{t}\right)=\exp \{-t \psi(z)\}$, where $\psi(z)$ is holomorphic for Re $z>0$ and continuous for $\operatorname{Re} z \geq 0$, with Re $\psi(z) \geq 0$. Moreover, $\psi(0)=0$, and $\psi^{\prime}(x)$ is completely monotone on $x>0$. The function $\psi(z)$ is called the Bernstein function.

The following two subordinator examples, involving the Gamma and Inverse Gaussian families respectively, yield closed form expressions.

$$
\begin{aligned}
& p_{t}(u)=\{\Gamma(\lambda t)\}^{-1} \sigma^{-\lambda t} u^{\lambda t-1} e^{-u / \sigma}, \quad \sigma, \lambda>0, \quad \mathcal{L}\left(p_{t}\right)=(1+\sigma z)^{-\lambda t}, \\
& p_{t}(u)=\left\{t e^{-t^{2} / 4 u}\right\} / \sqrt{4 \pi u^{3}}, \quad \mathcal{L}\left(p_{t}\right)=e^{-t \sqrt{z}} .
\end{aligned}
$$

The Inverse Gaussian is the special case $\beta=1 / 2$ of the important Lévy subordinator family of index $\beta$, where $0<\beta \leq 1$. For $\beta \neq 1 / 2$, this subordinator is not known in closed form and is defined implicitly by

$$
p_{t}(u)=\mathcal{L}^{-1}\left(e^{-t z^{\beta}}\right), \quad \operatorname{Re} z>0 .
$$

In the above, whenever multivalued Bernstein functions $\psi(z)$ appear, the particular branch of $\psi(z)$ such that $\operatorname{Re} \psi(z)>0$ on $\operatorname{Re} z>0$, is always understood.

Let $U(t)$ be the operator semigroup $e^{-t A}$ associated with the well-posed linear evolution equation $w_{t}=-A w, t>0, w(0)=f$, in a Banach space $B$. Bochner subordination involves randomizing the time variable in $U(t)$ as follows. Let $\left\{p_{t}(u)\right\}$ be a Bochner subordinator with Bernstein function $\psi(z)$, and define a new semigroup $T(t)$ by

$$
T(t) f=\int_{0}^{\infty} p_{t}(s) U(s) f d s, \quad f \in B
$$

Formally, $T(t)$ is the semigroup $e^{-t \psi(A)}$ associated with the well-posed linear evolution equation $w_{t}=-\psi(A) w, t>0, \quad w(0)=f$. See [12], [30], [32]. 
Next, let $A$ be a linear partial differential operator in the space variables in $R^{2}$, with constant coefficients. Let $\hat{h}(\xi, \eta, t)$ denote the Fourier transform of the Green's function for the linear evolution equation $w_{t}=-A w$. The Green's function $\hat{h}_{\psi}(\xi, \eta, t)$ for $w_{t}=-\psi(A) w$ can be obtained from $\hat{h}(\xi, \eta, t)$, using the composition law for subordinated characteristic functions [32, Chapter 6],

$$
\hat{h}_{\psi}(\xi, \eta, t)=\exp [-t \psi(-\log \{\hat{h}(\xi, \eta, 1)\})] .
$$

We may now apply the foregoing analysis to discover the diffusion equations associated with (8). We begin with the well-posed forward heat equation and its Gaussian Green's function

$$
\begin{aligned}
& w_{t}=a \Delta w, \quad t>0, \quad a>0, \\
& \hat{h}(\xi, \eta, t)=\exp \left(-t \sigma \rho^{2}\right), \quad \sigma=4 a \pi^{2}, \quad \rho=\sqrt{\xi^{2}+\eta^{2}} .
\end{aligned}
$$

Lévy subordination with $\psi(z)=z^{\beta}$ in (14), leads to a well-posed forward fractional diffusion equation, with a Lévy stable Green's function

$$
\begin{aligned}
& w_{t}=-\left[b(-\Delta)^{\beta}\right] w, \quad t>0, \quad b>0, \quad 0<\beta \leq 1, \\
& \hat{h}(\xi, \eta, t)=\exp \left(-t \delta \rho^{2 \beta}\right), \quad \delta=b\left(4 \pi^{2}\right)^{\beta}, \quad \rho=\sqrt{\xi^{2}+\eta^{2}} .
\end{aligned}
$$

Next, Gamma subordination with $\psi(z)=\lambda \log \{1+\sigma z\}$ in (15), results in a well-posed forward logarithmic diffusion equation, with a generalized Linnik Green's function

$$
\begin{aligned}
& w_{t}=-\left[\lambda \log \left\{1+c(-\Delta)^{\beta}\right\}\right] w, \quad t>0, \quad c>0, \quad 0<\beta \leq 1, \\
& \hat{h}(\xi, \eta, t)=\left(1+\gamma \rho^{2 \beta}\right)^{-\lambda t}, \quad \gamma=c\left(4 \pi^{2}\right)^{\beta}, \quad \rho=\sqrt{\xi^{2}+\eta^{2}} .
\end{aligned}
$$

The generalized Linnik process may also be viewed as resulting from a single subordination of Brownian motion with the Bernstein function $\psi(z)=\lambda \log \left(1+\sigma z^{\beta}\right)$.

Given the deconvolution problem $h(x, y) \otimes f(x, y)=g(x, y)$, with a known otf $\hat{h}(\xi, \eta)$ in the form of one the three types in (8), we view $\hat{h}(\xi, \eta)$ as the Green's function $\hat{h}(\xi, \eta, t)$ at time $t=1$, in the corresponding forward evolution equation $w_{t}=-L w$, in one of (14), (15), or (16). Deconvolution is mathematically equivalent to solving $w_{t}=-L w$ backwards in time, given the noisy blurred image $g(x, y)$ as data at time $t=1$. For $0<t<1, w(x, y, t)$ is a partially deblurred image. The fully deblurred image $f(x, y)$ is the solution at time $t=0$.

Such backwards continuation in diffusion equations is notoriously ill-posed. The SECB method, (see [25] for an up to date discussion), is a well-regularized continuation procedure that takes into account the presence of noise in $g(x, y)$ at $t=1$. With $n(x, y)$ as in (3), let constants $\epsilon, M$, be given such that

$$
\|w(0)\|_{2} \equiv\|f\|_{2} \leq M, \quad\|w(1)-g\|_{2} \equiv\|n\|_{2} \leq \epsilon, \quad \epsilon \ll M .
$$

For any constant $K>0$ such that $K \ll M / \epsilon$ define $s^{*}$ by

$$
s^{*}=\log \{M /(M-K \epsilon)\} / \log (M / \epsilon) .
$$

The slow evolution constraint applied to the backwards solution of $w_{t}=-L w$ requires that there exist a known small constant $K>0$ and a known fixed $s \gg s^{*}$, such that

$$
\|w(s)-w(0)\|_{2} \leq K \epsilon .
$$


Given the regularization parameters $K$ and $s$, the SECB solution is that initial value $w^{\dagger}(x, y, 0)=f^{\dagger}(x, y)$ which minimizes

$$
\|w(1)-g\|_{2}^{2}+K^{-2}\|w(s)-w(0)\|_{2}^{2},
$$

over all choices of initial values $w(x, y, 0)$ in $L^{2}$.

We may find the SECB deblurred image $f^{\dagger}(x, y)$ in closed form in the Fourier transform domain. We have, with $\bar{z}$ denoting the complex conjugate of $z$,

$$
\hat{f}^{\dagger}(\xi, \eta)=\frac{\overline{\hat{h}}(\xi, \eta, 1) \hat{g}(\xi, \eta)}{|\hat{h}(\xi, \eta, 1)|^{2}+K^{-2}\left|1-\hat{h}^{s}(\xi, \eta, 1)\right|^{2}},
$$

leading to $f^{\dagger}(x, y)$ upon inverse transforming. Of particular interest is the partially deblurred image $w^{\dagger}(x, y, t)$ defined by

$$
\hat{w}^{\dagger}(\xi, \eta, t)=\frac{\hat{h}(\xi, \eta, t) \overline{\hat{h}}(\xi, \eta, 1) \hat{g}(\xi, \eta)}{|\hat{h}(\xi, \eta, 1)|^{2}+K^{-2}\left|1-\hat{h}^{s}(\xi, \eta, 1)\right|^{2}}, \quad 0 \leq t<1 .
$$

This can be efficiently implemented using FFT algorithms.

4. Blind deconvolution by marching backwards in time. As indicated in Section 2, given an image $g(x, y)$, we seek to identify the system otf by a least squares fit to the data $\log |\hat{g}(\xi, 0)|$ with a suitable monotone decreasing function $p(\xi)$ with $p(0)=0$, as in Figure 1. The function $p(\rho)$ is chosen so that $\exp \{p(\rho)\}$ is one of the three characteristic function types in (8). We then define

$$
\hat{h}(\xi, \eta, t)=\exp \{t p(\rho)\}, \quad 0 \leq t \leq 1, \quad \rho=\sqrt{\xi^{2}+\eta^{2}} .
$$

where $\hat{h}(\xi, \eta, t)$ is the Green's function for the corresponding diffusion equation in one of (14), (15), or (16). Using this in (22), together with $g(x, y)$ as data at $t=1$, great benefit derives from the ability to perform the deconvolution in slow motion by marching backwards in time in the diffusion equation $w_{t}=-L w$. Recall that from (7), the system otf satisfies $\log \hat{h}(\rho) \sim(1-c) p(\rho), \rho \gg 1$, for some unknown positive constant $c<1$. Continuation all the way to $t=0$ in $(22)$, with $\hat{h}(\xi, \eta, t)$ as in (23), is equivalent to falsely setting $c=0$ in (7). This necessarily produces oversharpening. Displaying the evolution of $w^{\dagger}(x, y, t)$ as $t$ decreases from 1 to 0 , allows for monitoring the deblurring process. At first, the partially deblurred images $w^{\dagger}(x, y, t)$ become sharper and slightly noisier as $t$ decreases. However, as expected, ringing, noise, and other artifacts begin to appear, indicating that continuation has proceeded too far. Diagnostic statistical information about $w^{\dagger}(x, y, t)$ can also be calculated for selected values of $t$, as $t$ decreases. Of particular interest are the discrete $L^{1}$ norm $\left\|w^{\dagger}(., t)\right\|_{1}$, and the discrete $T V$ norm $\left\|\nabla w^{\dagger}(., t)\right\|_{1}$. In well-behaved deconvolution, image flux is conserved, and $\left\|w^{\dagger}(., t)\right\|_{1}$ remains constant as $t \downarrow 0$. At the same time, $\left\|\nabla w^{\dagger}(., t)\right\|_{1}$ increases monotonically, reflecting the gradual sharpening of edges and other localized singularities. Such visual and statistical monitoring enable selection of an optimal image $w^{\dagger}(x, y, t)$, which is found at some $\bar{t}>0$. Terminating continuation at $\bar{t}>0$, is equivalent to redefining $p(\rho)$ in $(23)$ to be $\bar{p}(\rho)=(1-\bar{t}) p(\rho)$, and then selecting the image at $t=0$ as optimal. Clearly, $\bar{t}$ corresponds to the unknown constant $c$ in (7).

In practice, the true system otf is seldom found, and the image $L^{1}$ norm may show a modest increase as $t$ decreases from $t=1$ to $t=\bar{t}$. Conservation of $L^{1}$ norm 


\section{Least squares fit in M51}
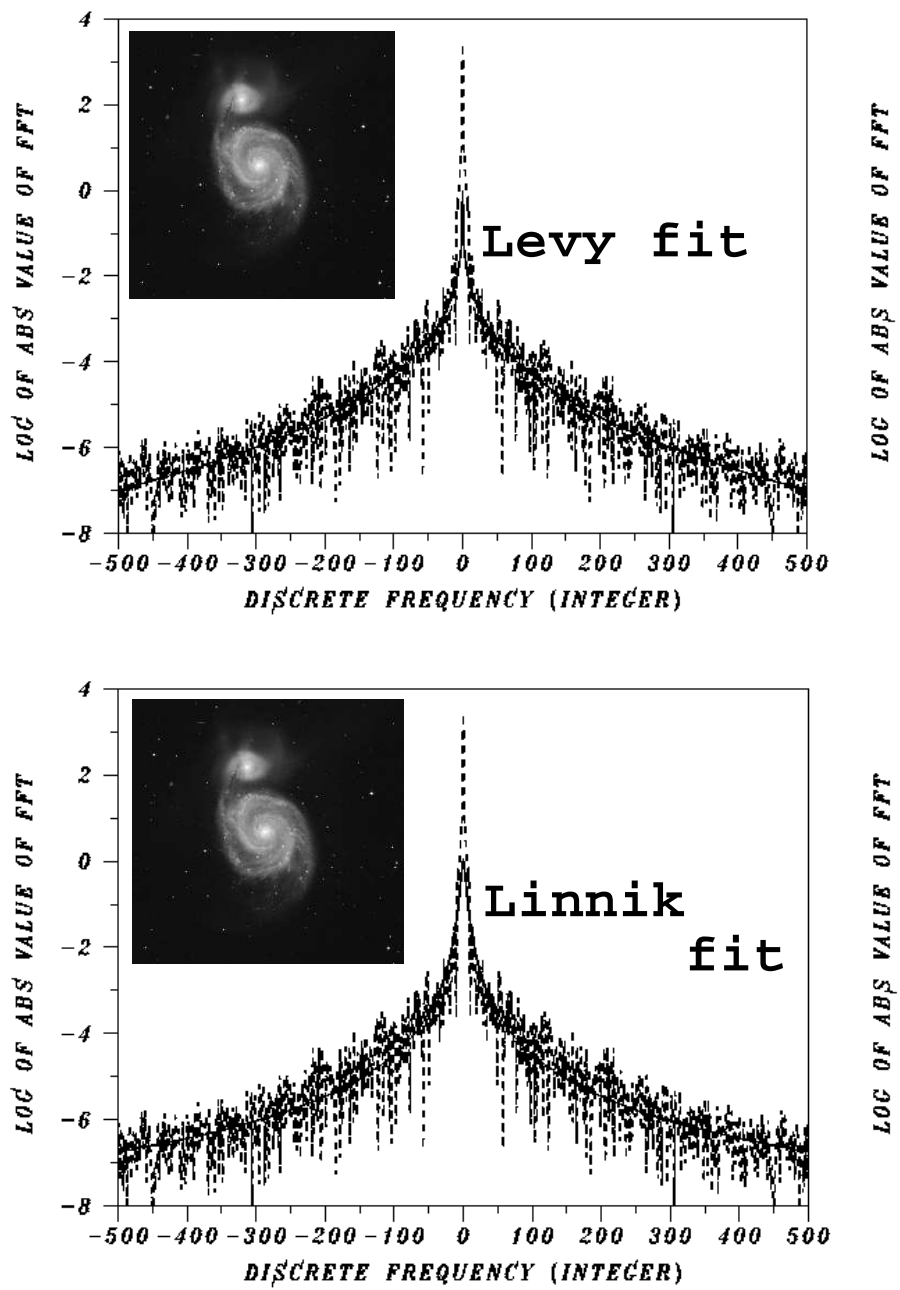

\section{Behavior near the crigin}

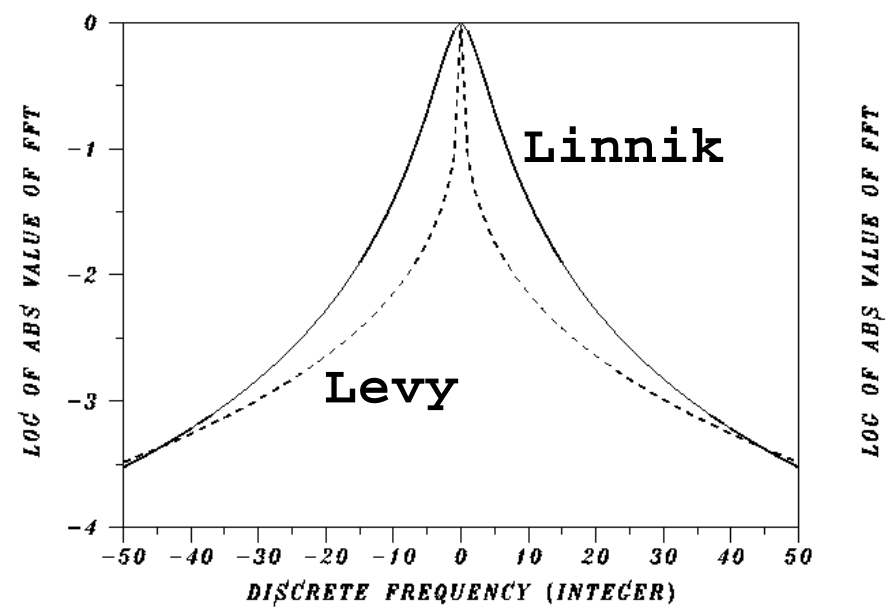

FiG. 2. Blurred M51 image data $\log |\hat{g}(\xi, 0)|$ can be well-fitted with a Lévy stable otf, as well as with a generalized Linnik otf. Both otfs have similar behavior away from the origin, but differ fundamentally near the origin. This difference will play a crucial role. 


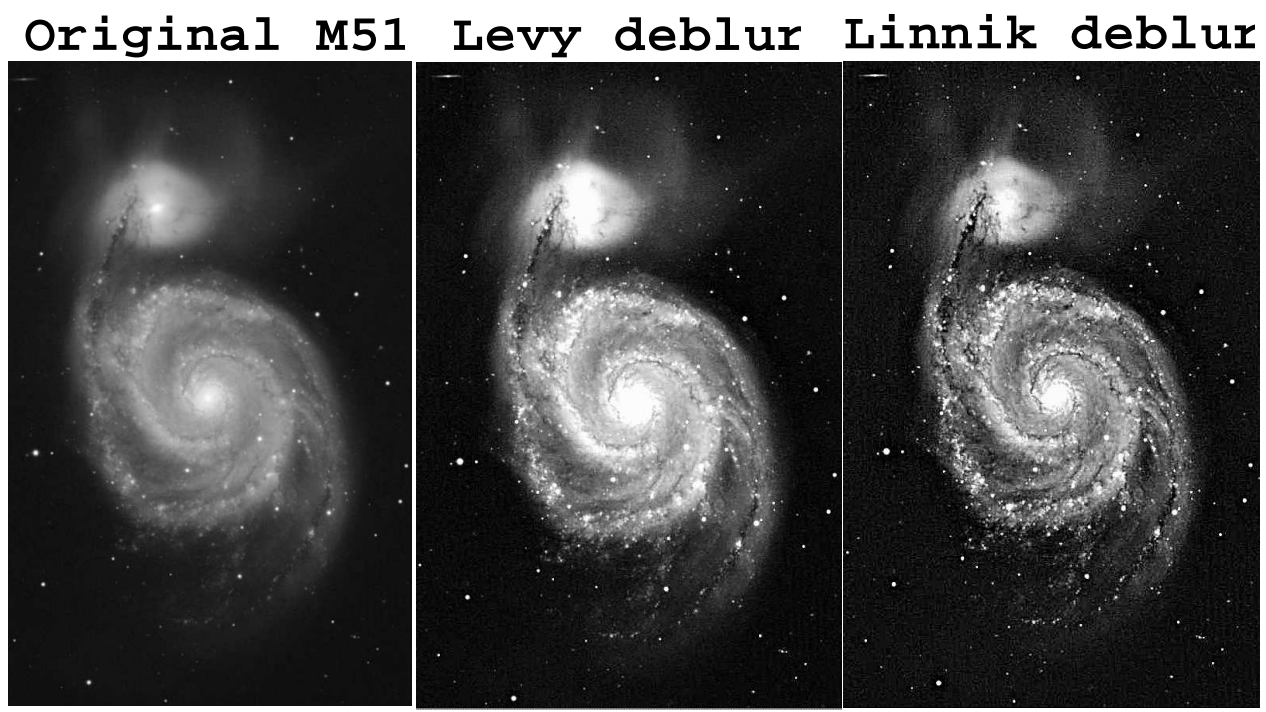

\section{Lipschitz exponents in Whirlpool imag}

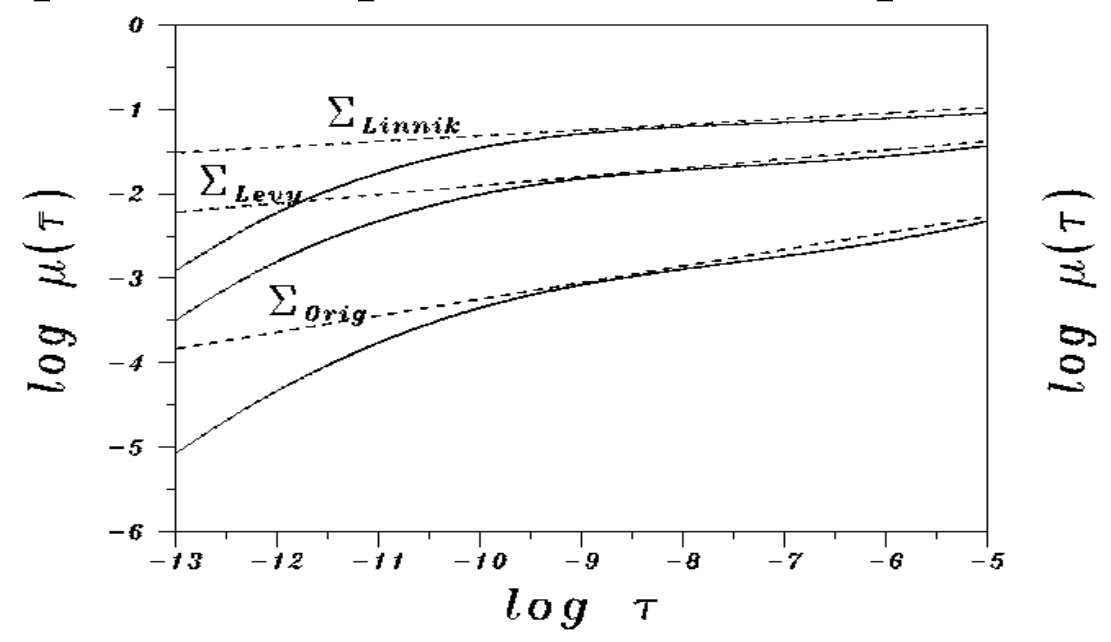

FIG. 3. Logarithmic diffusion equation produces higher quality reconstruction of Whirlpool galaxy image than does fractional diffusion equation. Such differences in quality are reflected in the smallness of the image Lipschitz exponent, which equals twice the slope of the corresponding $\Sigma$ line. Here, Original image has Lip $\alpha=0.39$, Lévy image has Lip $\alpha=0.21$, and Linnik image has Lip $\alpha=0.13$.

in $w^{\dagger}(x, y, t)$ can be enforced for any desired $t$, by rescaling $w^{\dagger}(x, y, t)$ to the value $\|g\|_{1}$. Moreover, as emphasized in [7, Figure 1], given a blurred $g(x, y)$, there may be infinitely many distinct otfs $\hat{h}(\xi, \eta)$ that can competently deblur that image.

5. Logarithmic vs fractional diffusion in Whirlpool galaxy image. We shall now demonstrate the significance of the preceding developments by applying them to sharpen real blurred images with unknown blurs, subject to real but un- 
known noise processes. Our first example is a $1024 \times 1024$ pixels image $g(x, y)$ of the Whirlpool galaxy (M51), taken by Travis Rector and Monica Ramirez at the Kitt Peak National Observatory (NOAO/AURA/NSF), (National Optical Astronomy Observatory/Associated Universities for Research in Astronomy/National Science Foundation).

In Figure 2, we examine the data $\log |\hat{g}(\xi, 0)|$. With $\rho=\sqrt{\xi^{2}+\eta^{2}}$, we first consider a Lévy stable fit using $\hat{h}(\rho)=\exp \left(-\delta \rho^{2 \beta}\right), \quad \delta>0,0<\beta \leq 1$. While a Gaussian otf $(\beta=1.0)$ is an allowed possiblity, the monotone convexity property in $\log |\hat{g}(\xi, 0)|$ results in a least squares fit with $\delta=1.07, \beta=0.15$. Next, with $w(x, y, 1)=g(x, y)$, we solve the fractional diffusion equation $w_{t}=-\left[b(-\Delta)^{\beta}\right] w, \quad 0 \leq t \leq 1, \quad b=$ $\delta\left(4 \pi^{2}\right)^{-\beta}$, backwards in time, using an FFT implementation of (22). With the regularization parameters $K=40.0, s=0.001$, the optimal image was found at $\bar{t}=0.75$. The $L^{1}$ norm was conserved on $\bar{t} \leq t \leq 1.0$, while the $T V$ norm increased almost fourfold. The reconstructed image is the middle image at the top of Figure 3. The value $\beta=0.15$ for the Lévy exponent, is much lower than what is typically found in most physical applications of Lévy stable laws.

The analysis in Section 2 emphasizes the behavior of $\hat{h}(\rho)$ away from the origin, and makes it plausible that the system otf might be identifiable from that behavior. For blurred imagery with monotone convex Fourier decay, low exponent Lévy stable laws are natural candidate otfs in blind deconvolution. The fundamental observation of this paper is that there exist otfs with behavior away from the origin that is almost identical to that in low exponent Lévy stable laws, yet with behavior near the origin that is almost Gaussian. Indeed, consider a generalized Linnik fit to the same $M 51$ data $\log |\hat{g}(\xi, 0)|$, using $\hat{h}(\rho)=\left(1+\gamma \rho^{2 \sigma}\right)^{-\lambda}, \quad \gamma, \lambda>0, \quad 0<\sigma \leq 1$, as shown in Figure 2. This results in $\sigma=0.86, \gamma=0.09, \lambda=0.821$. The bottom drawing in Figure 2 illustrates the major difference between Levy and Linnik otfs near the origin. We next solve the backwards in time logarithmic diffusion problem, $w_{t}=-\left[\lambda \log \left\{1+c(-\Delta)^{\sigma}\right\}\right] w, \quad c=\gamma\left(4 \pi^{2}\right)^{-\sigma}, 0 \leq t \leq 1, \quad w(x, y, 1)=g(x, y)$. Here, with $K=40.0, s=0.001$ in (22), we now find the optimal image at $\bar{t}=0.65$. Again the $L^{1}$ norm was conserved on $\bar{t} \leq t \leq 1.0$, but the $T V$ norm increased almost sevenfold. The reconstructed image is the rightmost image at the top of Figure 3.

Clearly, while the Lévy deblurred image significantly sharpens the original, the Linnik deblurred image provides higher quality reconstructions of the spiral arms, dustlanes, and galactic cores. The image Lipschitz exponent, discussed below, is a valuable image metrology tool that can measure the extent of fine structure recovery in deblurring, and can quantify any improvement produced using logarithmic rather than fractional diffusion equations. This tool will be used throughout this paper.

6. Fine structure recovery and image Lipschitz exponents. An image $f(x, y)$ has $L^{1}$ Lipschitz exponent $\alpha$, if and only if

$$
\int_{\mathbf{R}^{2}}\left|f\left(x+h_{1}, y+h_{2}\right)-f(x, y)\right| d x d y \leq \text { Const }|h|^{\alpha}, \quad|h| \rightarrow 0,
$$

where $|h|=\left(h_{1}^{2}+h_{2}^{2}\right)^{1 / 2}$, and $\alpha$ is fixed with $0<\alpha \leq 1$. Most images are not smoothly differentiable functions of $x$ and $y$, but display edges, localized sharp features, and other significant fine scale details or texture. The Lipschitz exponent measures the fine structure content of an image, provided that image is relatively noise free. The value of $\alpha$ decreases with increasing fine structure. An image that is of bounded variation, or smoother, has $\alpha=1$. Most natural images have $\alpha<0.6$, and are not of bounded variation. Images of starfields, galaxies, and clusters of galaxies, often have $\alpha<0.3$. 
In [8], [13], an effective method for estimating image Lipschitz exponents is developed, based on a major theorem in [34]. For fixed $\tau>0$, define the linear operator $G^{\tau}$ by means of the Fourier series

$$
G^{\tau} f=\sum_{\xi, \eta=-\infty}^{\infty} \exp \left\{-\tau\left(\xi^{2}+\eta^{2}\right)\right\} \hat{f}(\xi, \eta) \exp \{2 \pi i(x \xi+y \eta)\}
$$

Let $\mu(\tau)=\left\|G^{\tau} f-f\right\|_{1} /\|f\|_{1}$, be the $L^{1}$ relative error in approximating $f$ with $G^{\tau} f$. An image $f(x, y)$ has Lipschitz exponent $\alpha$ if and only if $\mu(\tau)=O\left(\tau^{\alpha / 2}\right)$ as $\tau \downarrow 0$. Using FFT algorithms, (25) can be evaluated for each fixed $\tau_{n}>0$ in a sequence $\left\{\tau_{n}\right\}$ tending to zero, together with $\mu\left(\tau_{n}\right)$. By plotting $\mu\left(\tau_{n}\right)$ vs $\tau_{n}$ on a log-log scale, positive constants $C$ and $\alpha$ can be located such that $\mu(\tau) \leq C \tau^{\alpha / 2}$ as $\tau \downarrow 0$.

The three solid curves in the bottom drawing in Figure 3, are plots of $\mu(\tau)$ vs $\tau$ for each image in Figure 3. Each plot is majorized by a dashed straight line $\Sigma$. The Lipschitz exponent $\alpha$ of each image is equal to twice the slope of the corresponding $\Sigma$ line. Accordingly, the original M51 image has Lip $\alpha=0.39$. The Lévy deblurred image has Lip $\alpha=0.21$, indicating significant sharpening of the original. However, the Linnik deblurred image has Lip $\alpha=0.13$, a striking improvement.

7. Tail behavior in physical space and mysterious Lévy exponents. The improved reconstruction of M51 using the Linnik otf, is directly traceable to the difference in behavior near the origin in the bottom drawing in Figure 2. As can be inferred from that drawing, selecting the Lévy otf rather than the Linnik otf, implicitly declares the low frequencies in the blurred image $g(x, y)$ to be significantly more attenuated than would be implied by the Linnik otf. Consequently, in deblurring with the fractional diffusion equation, such low frequencies are necessarily amplified to a larger extent than would occur using the logarithmic diffusion equation. Such spurious overamplification causes saturation and loss of resolution in the galactic cores and spiral arms in the Lévy image. To mitigate this effect, the exit point $\bar{t}$ in the Lévy case must generally be chosen larger than in the Linnik case, which leads to less sharpening. As shown in Figure 4, such Lévy image saturation phenomena near the galactic cores are fairly common. As will be seen below, Linnik otf behavior near the origin plays a beneficial role in all the blind deconvolution experiments in this paper.

In physical $(x, y)$ space, both the isotropic 2D Lévy stable and generalized Linnik probability densities are heavy-tailed densities. However, although the corresponding characteristic functions can have the almost identical high frequency behavior shown in Figure 2, the tail behavior in physical $(x, y)$ space is distinctly different. Indeed, such tail behavior is directly related to the smoothness of the characteristic function near the origin in Fourier $(\xi, \eta)$ space. The following important results may be found in $[26]$.

$$
\begin{gathered}
\hat{h}_{\text {Levy }}(\xi, \eta)=\exp \left(-\delta \rho^{2 \beta}\right), \quad \delta>0, \quad 0<\beta<1, \quad \rho=\sqrt{\left(\xi^{2}+\eta^{2}\right)}, \\
h_{\text {Levy }}(x, y)=O\left(R^{-2-2 \beta}\right), \quad R \uparrow \infty, \quad R=\sqrt{\left(x^{2}+y^{2}\right)} . \\
\hat{h}_{\text {Linn }}(\xi, \eta)=\left(1+\gamma \rho^{2 \sigma}\right)^{-\lambda}, \quad \gamma, \lambda>0, \quad 0<\sigma<1, \quad \rho=\sqrt{\left(\xi^{2}+\eta^{2}\right)}, \\
h_{\text {Linn }}(x, y)=O\left(R^{-2-2 \sigma}\right), \quad R \uparrow \infty, \quad R=\sqrt{\left(x^{2}+y^{2}\right)} .
\end{gathered}
$$




\section{Orig $\mathrm{M} 61$}

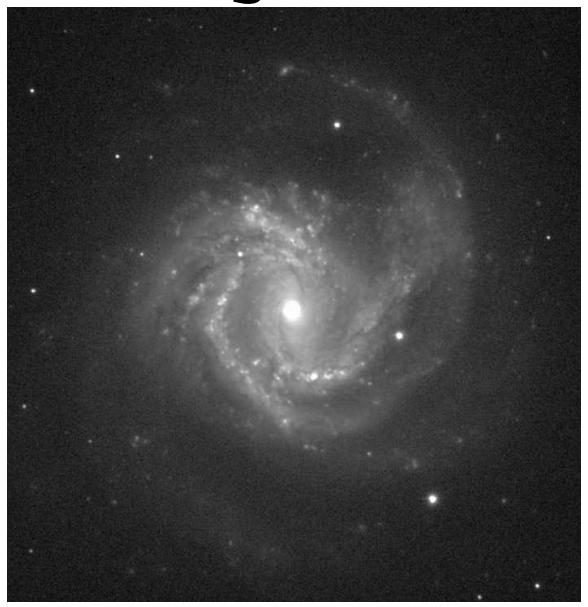

Levy deblur

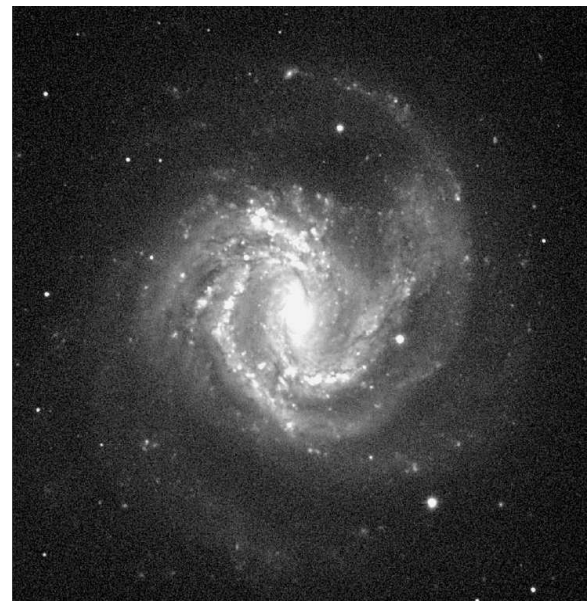

Linnik deblur

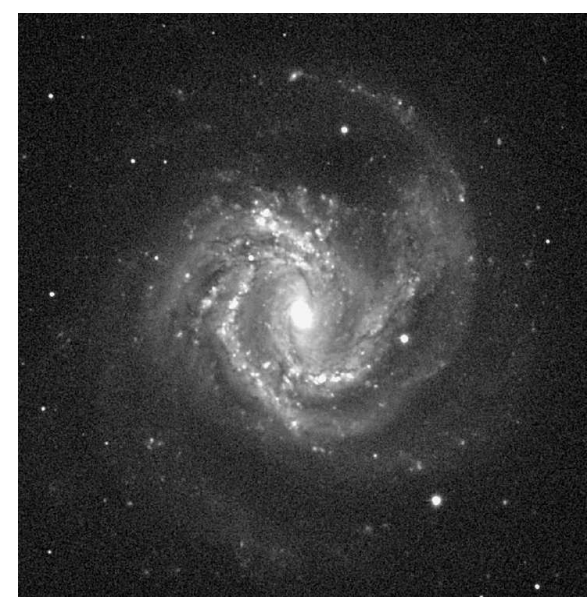

Orig NGC3310

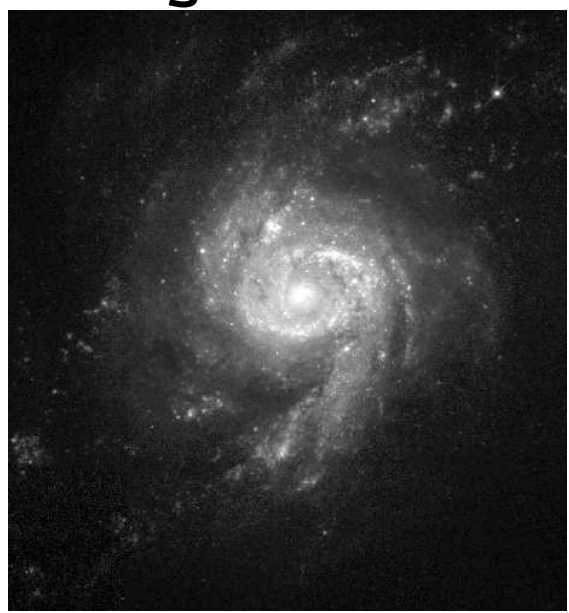

\section{Levy deblur}

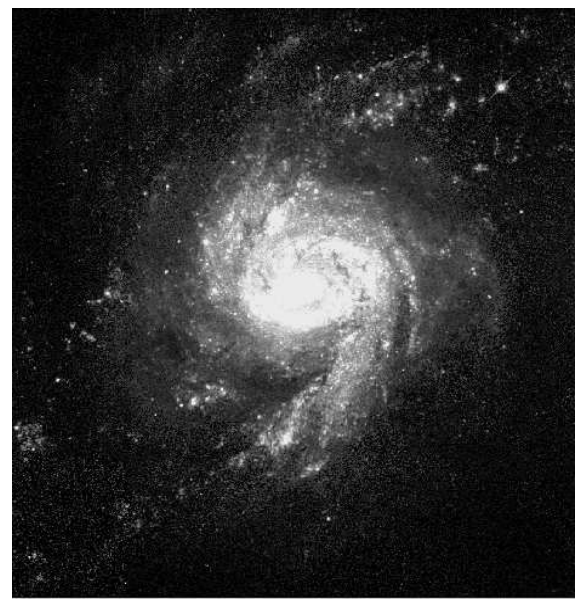

Linnik deblur

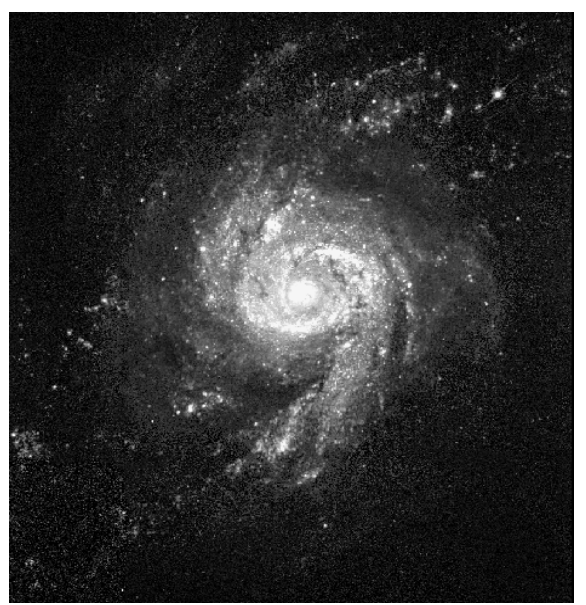

FIG. 4. Fractional diffusion processing generally causes saturation and loss of resolution near galactic cores, due to the behavior of Lévy stable otfs near the origin, as shown in Figure 2. The use of logarithmic diffusion avoids this difficulty. 
Applied to the M51 image in Figure 3, where $\delta=1.07, \beta=0.15, \sigma=0.86, \gamma=0.09$, and $\lambda=0.821$, this leads to

$$
h_{\text {Levy }}(x, y)=O\left(R^{-2.3}\right), \quad h_{\text {Linn }}(x, y)=O\left(R^{-3.72}\right), \quad R \uparrow \infty .
$$

Thus, the Linnik density has a much thinner tail than the Lévy density in physical space. In summary, in the Whirlpool galaxy image in Figure 3, the successful generalized Linnik point spread function has the tail behavior of a Lévy stable density with exponent $\beta=0.86$ in physical $(x, y)$ space, but the high-frequency behavior of a stable density with $\beta=0.15$ in Fourier $(\xi, \eta)$ space. Similar behavior characterizes the successful Linnik otfs in other blind deconvolution experiments in this paper. This result resolves the unexplained appearance of exceptionally low Lévy exponents in the detected successful Lévy otfs in [10] and [11]. Clearly, such low exponents are not physically meaningful, but stem from choosing Lévy stable otfs, rather than generalized Linnik otfs, to match the blurred image data in [10] and [11].

8. Logarithmic diffusion and Hubble space telescope imagery. It is remarkable that the seemingly naive methodology developed in the previous sections can be useful in improving Hubble imagery. However, in many cases, faint background objects can be made more visible, and the structure of foreground objects can become more clearly defined. This appears to be possible for the third-generation instrument known as the Advanced Camera for Surveys (ACS), recently repaired in May 2009, as well as for the 'workhorse' Wide Field and Planetary Camera 2, (WFPC2), replaced with WFPC3 in May 2009. Access to the on-line version of this paper will be helpful in the subsequent discussion. Credit for the three images to be deconvolved below includes NASA, (National Aeronautics and Space Administration), ESA, (European Space Agency), the Hubble Heritage Team (STScI/AURA), (Space Telescope Science Institute/Association of Universities for Research in Astronomy), A. Riess (STScI), and K. Noll (STScI).

1. Pinwheel galaxy NGC 1309. The example at the top of Figure 5 is a February 2006 Hubble telescope image of the spiral galaxy NGC 1309. The image was taken with Hubble's most powerful camera, the Advanced Camera for Surveys. NASA describes this image as featuring bright bluish clusters of star formation, together with dust lanes spiraling into a yellowish central core of older-population stars. The image is complemented by far-off background galaxies. This galaxy is also home to a supernova that can help astronomers measure the expansion rate of the universe.

Considerable enhancement of these features is possible using logarithmic diffusion. The color image was broken up into its constituent red, green, and blue component images (RGB), and each component was treated in turn. For each component, $\log |\hat{g}(\xi, 0)|$ was best-fitted with the generalized Linnik otf expression $\hat{h}(\rho)=$ $\left(1+\gamma \rho^{2 \sigma}\right)^{-\lambda}, \quad \gamma, \lambda>0, \quad 0<\sigma \leq 1$. For the blue component, this results in $\gamma=0.174, \lambda=0.653$, and $\sigma=0.88$. Otfs for the other two components were found to differ only slightly from the blue component otf. The latter was therefore used for all three components. We next solve the backwards in time logarithmic diffusion problem, $w_{t}=-\left[\lambda \log \left\{1+c(-\Delta)^{\sigma}\right\}\right] w, \quad c=\gamma\left(4 \pi^{2}\right)^{-\sigma}, 0 \leq t \leq 1, \quad w(x, y, 1)=g(x, y)$, using (22). In all three cases, with the regularization parameters $K=40.0, s=0.001$, and with $\bar{t}=0.7$, the $L^{1}$ norm was conserved on $1 \leq t \leq \bar{t}$, while the $T V$ norm increased fivefold.

The Linnik deblurred image is shown at the bottom of Figure 5. At the outskirts of that image, several far-off galaxies have become more visible, and the scythe-shaped 


\section{Original HST NGC130}

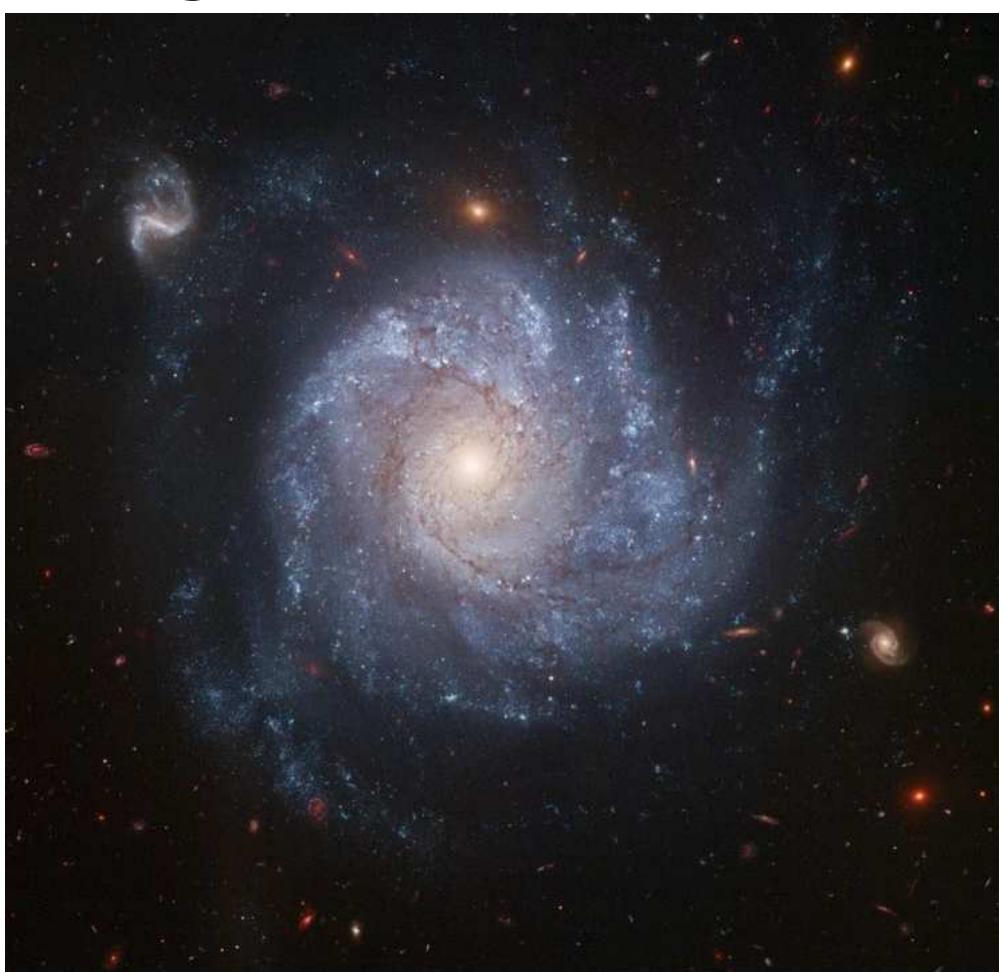

\section{Linnik deblur}

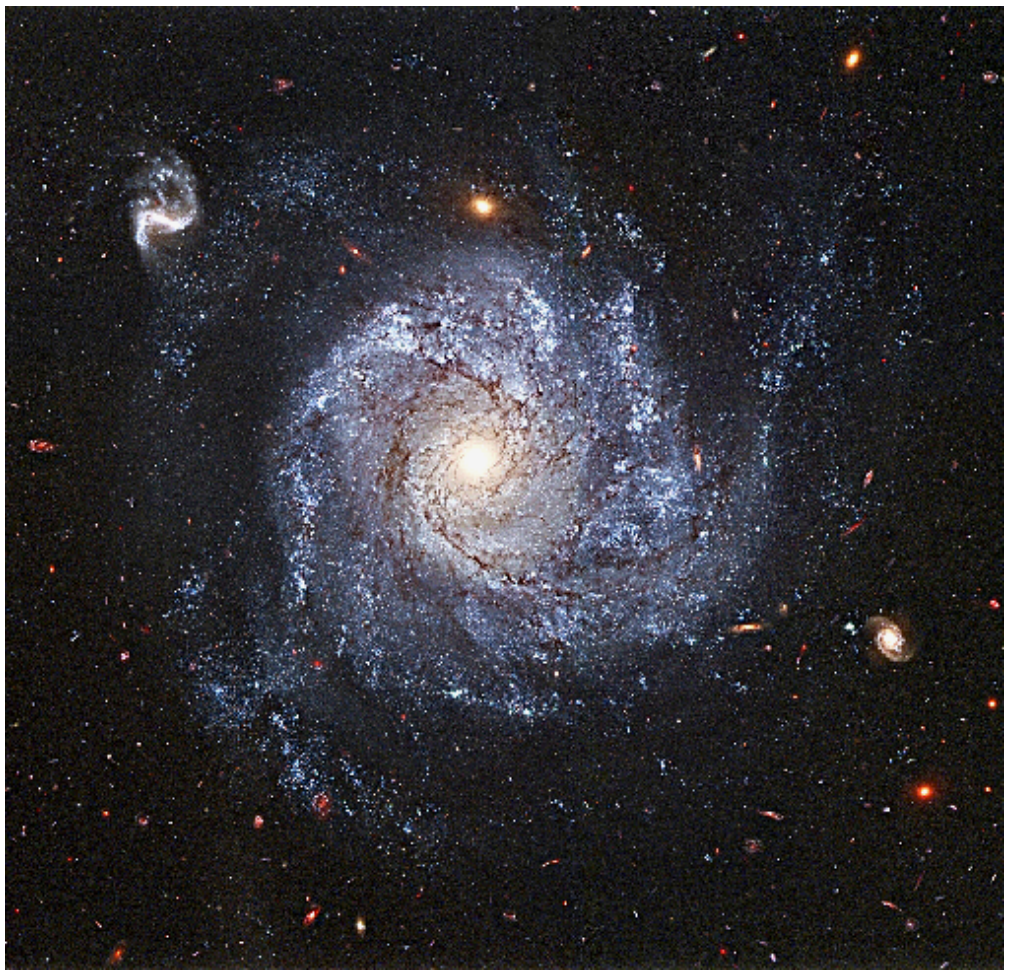

FIG. 5. Successful blind deconvolution of Hubble space telescope NGC 1309 image. Original blue component image has Lip $\alpha=0.25$. Linnik blue component has Lip $\alpha=0.09$. 


\section{Original HST NGC60\$}

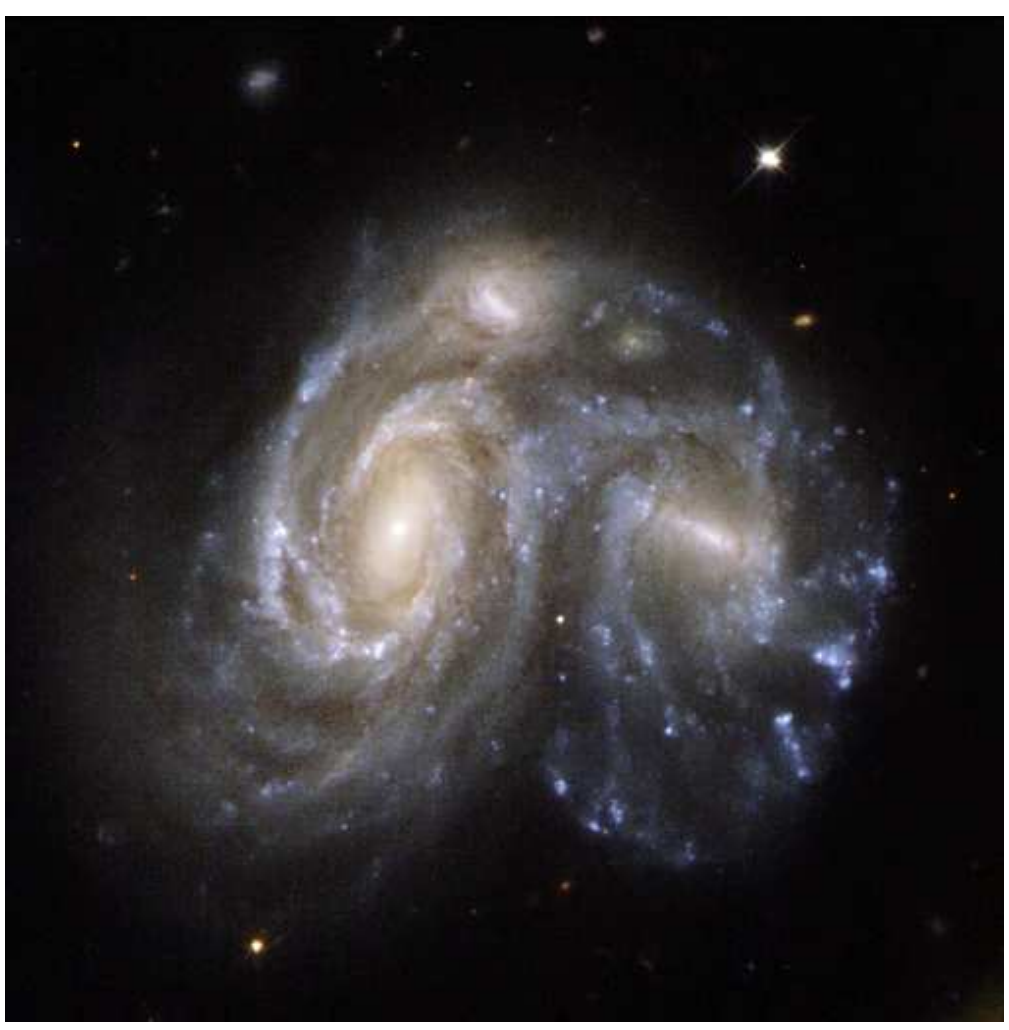

\section{Linnik deblur}

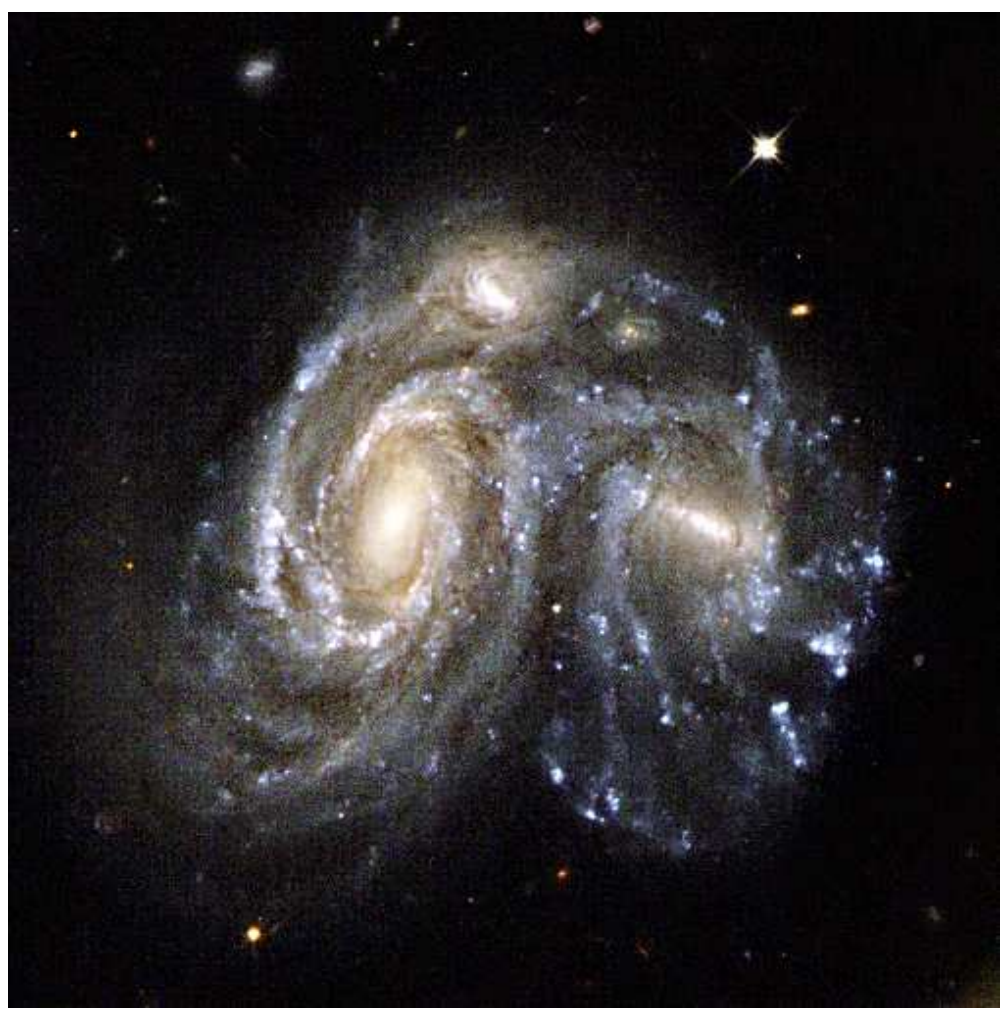

FIG. 6. Successful blind deconvolution of Hubble space telescope NGC 6050 image. Original blue component image has Lip $\alpha=0.28$. Linnik blue component has Lip $\alpha=0.15$. 


\section{Original HST NGC33D}

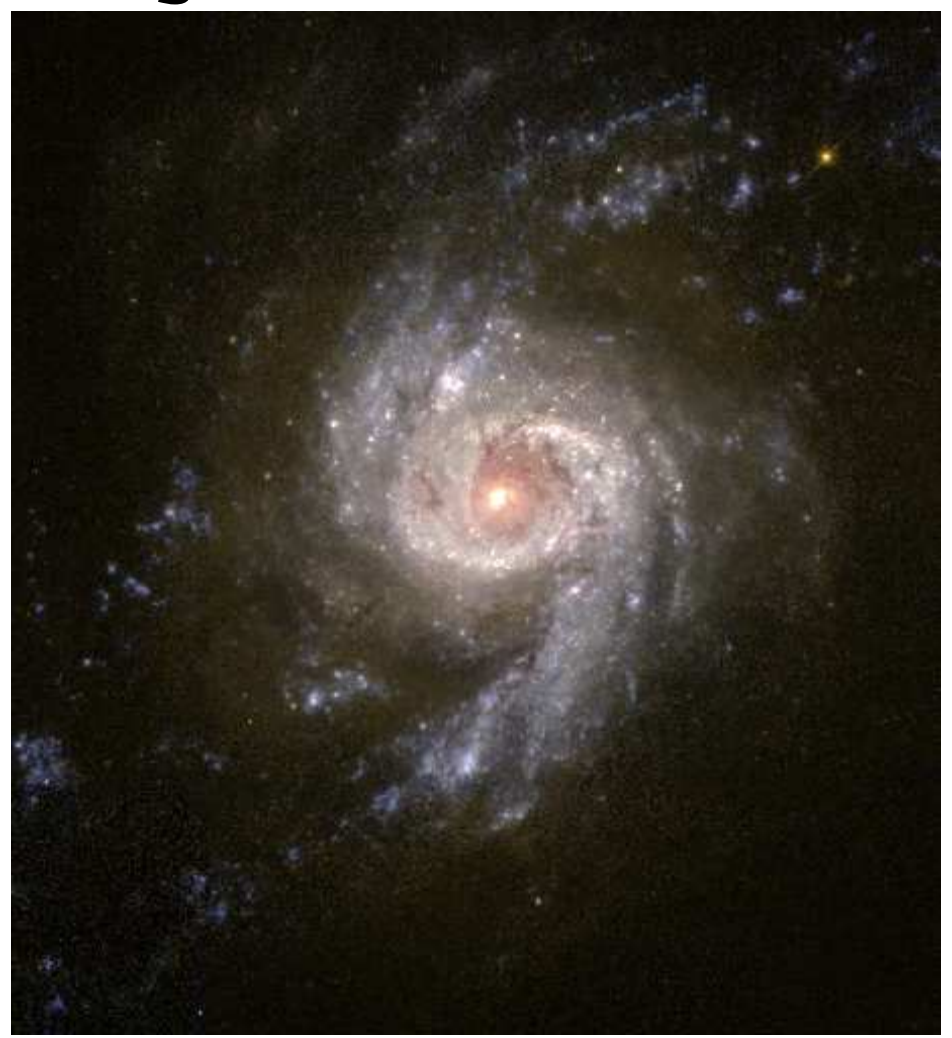

\section{Iinnik deblur}

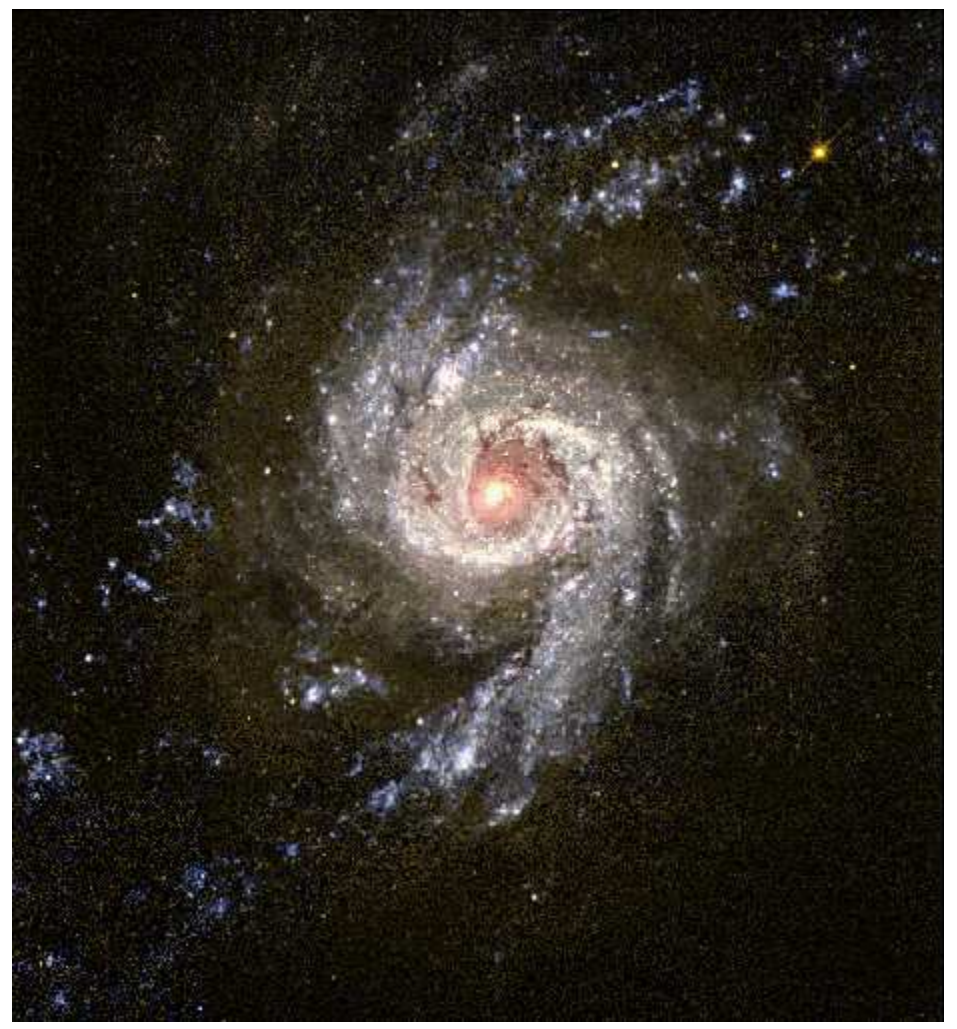

FIG. 7. Successful blind deconvolution of Hubble space telescope NGC 3310 image. Original red component image has Lip $\alpha=0.26$. Linnik red component has Lip $\alpha=0.14$. 


\section{Original SEN.}

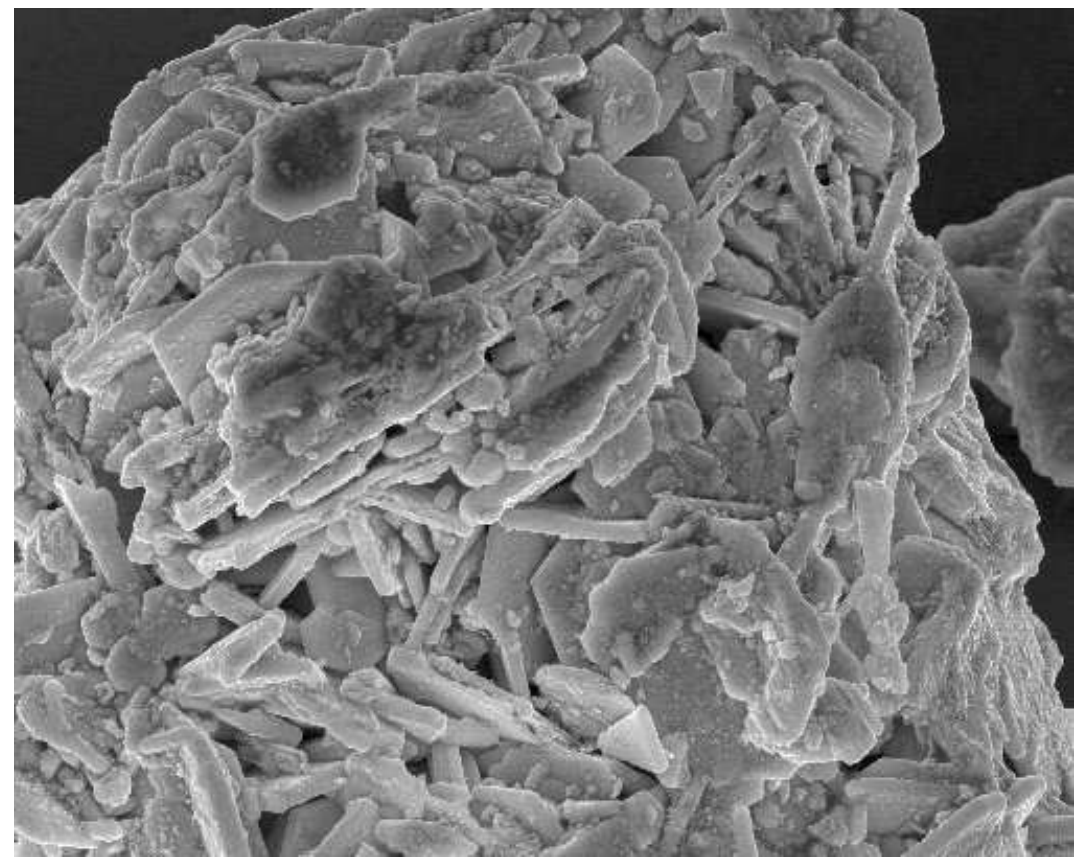

\section{Linnik deblur}

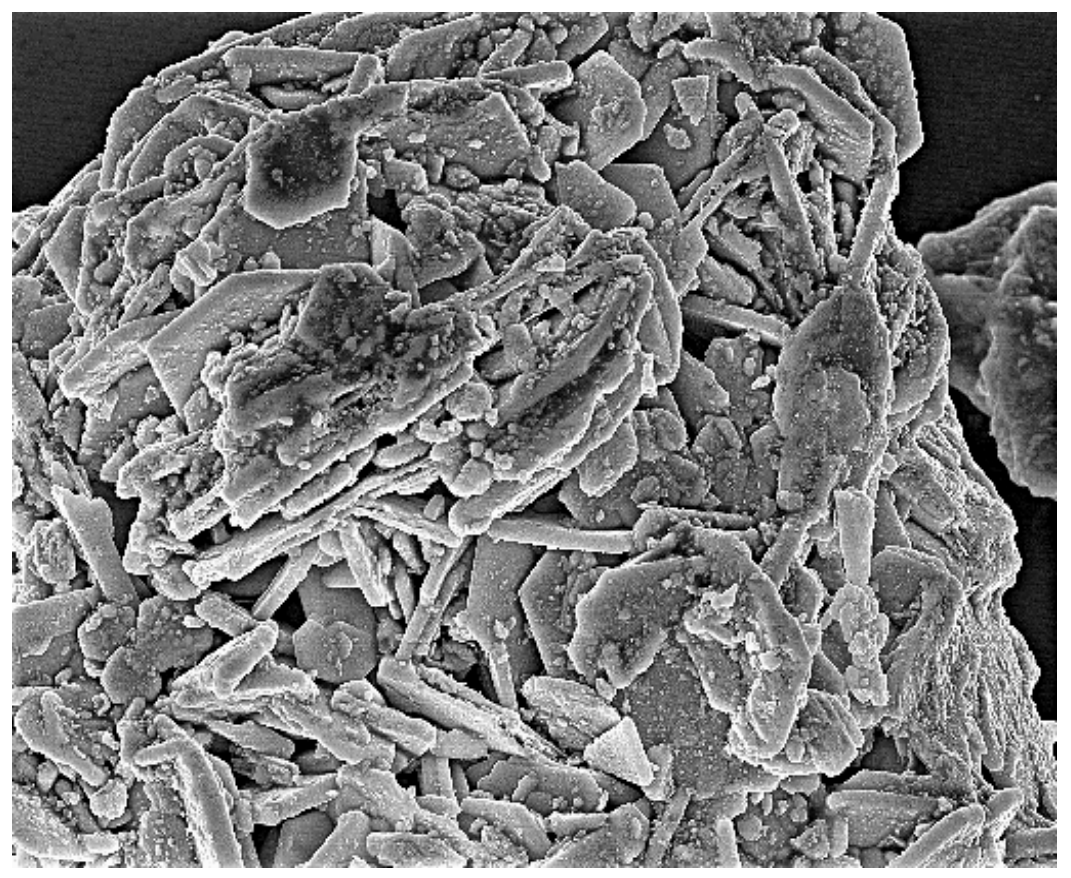

FIG. 8. Blind deconvolution of scanning electron micrograph of complex crystalline structure recovers numerous surface particles and other fine detail. Original image has Lip $\alpha=0.37$. Linnik image has Lip $\alpha=0.20$. 


\section{Original SEN.}

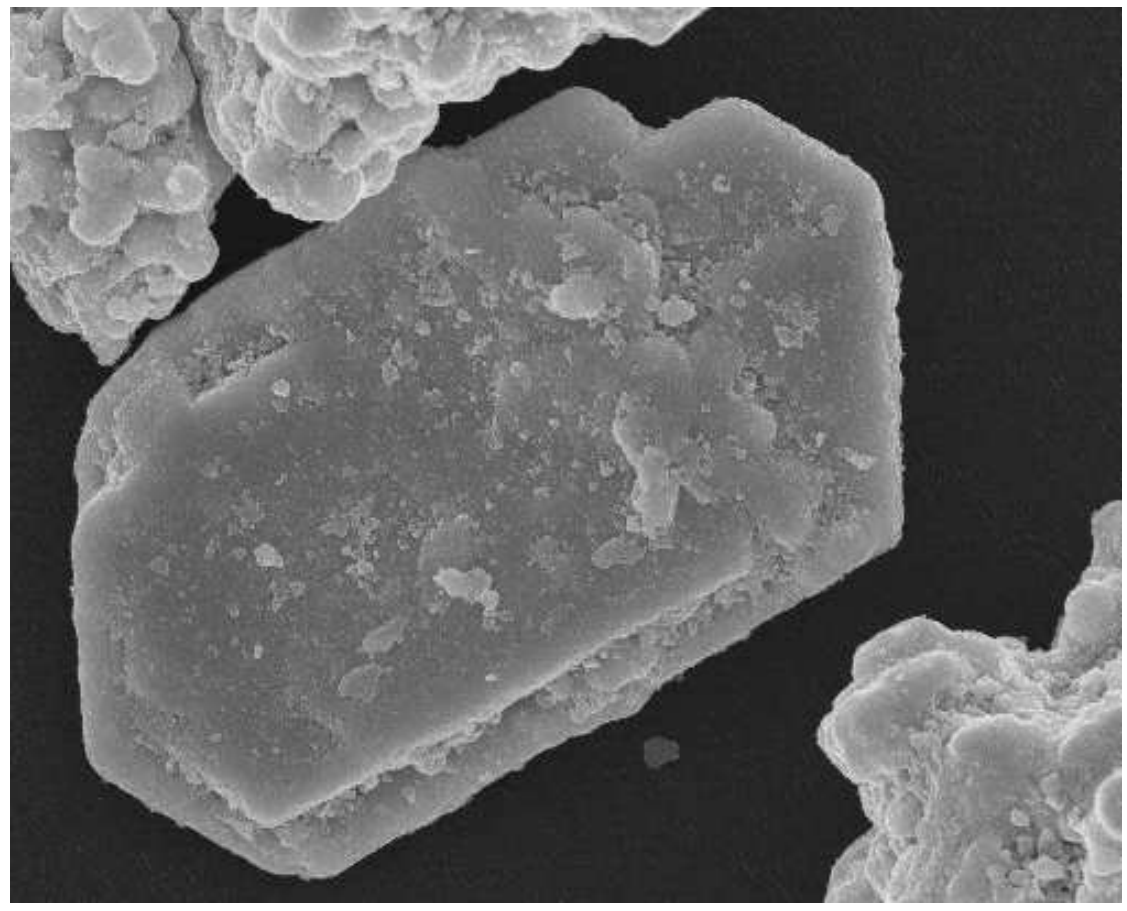

\section{Linnik deblur}

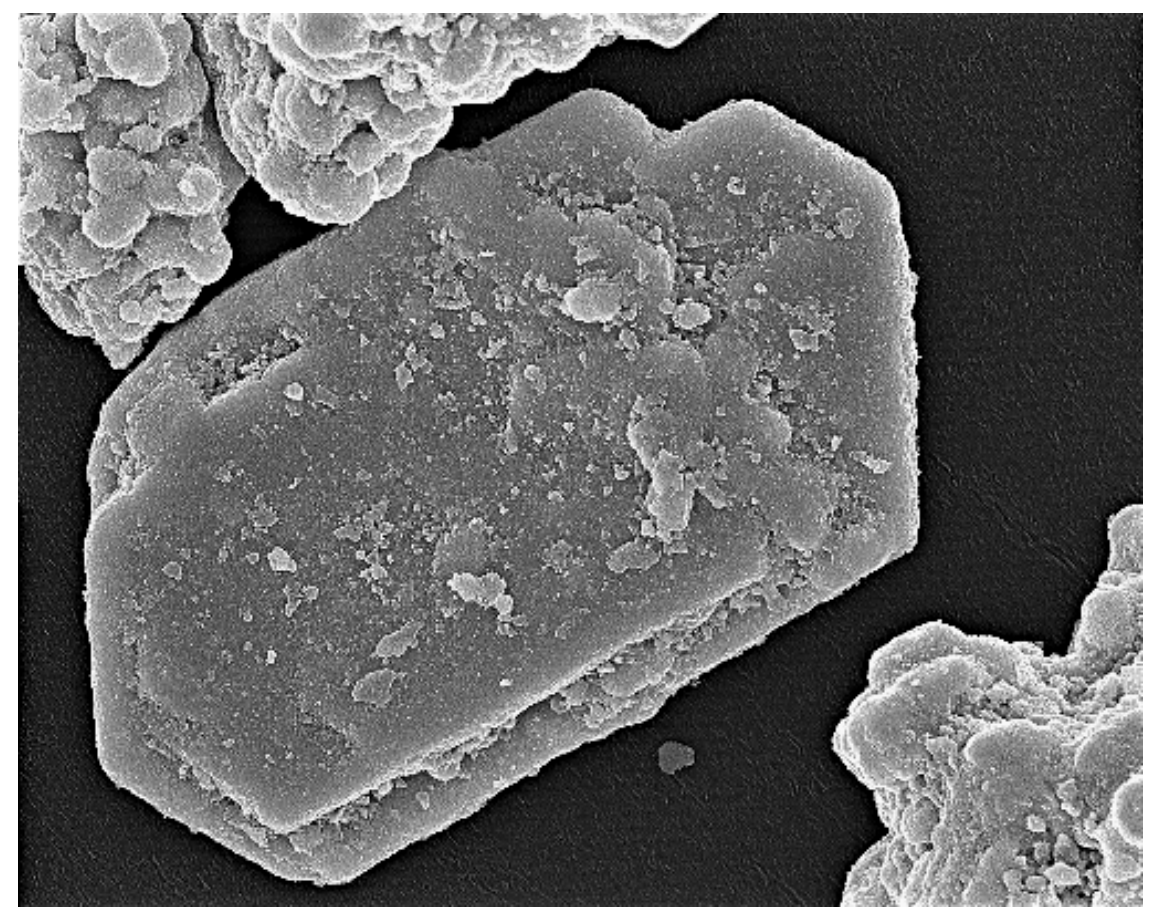

FIG. 9. Blind deconvolution of scanning electron micrograph of slab like crystalline structure recovers fine scale detail. Note improvement in corner structures. Original image has Lip $\alpha=0.48$. Linnik image has Lip $\alpha=0.25$. 
structure at eleven o'clock is now brighter and more clearly defined. Previously barely visible outer spiral arms have been recovered, along with a multitude of previously faint stars. Within the galaxy's main body, the bluish star clusters along the spiral arms, and the dust lanes, are now much better resolved.

Evidently, significant fine-structure reconstruction was achieved, and this is reflected in Lipschitz exponents. For the blue component, the original image has Lip $\alpha=0.25$, while the Linnik image has Lip $\alpha=0.09$. Lévy deblurring of the blue component required termination at $\bar{t}=0.8$, and displayed loss of resolution due to saturation. That image had Lip $\alpha=0.12$.

2. Colliding galaxies NGC 6050. The example at the top of Figure 6 involves a spectacular collision between two spiral galaxies. That image is part of a large collection of similar images released by NASA in April 2008. Acquired using Hubble's Wide Field and Planetary Camera 2, the image represents a rarely observed snapshot of a galactic merger thought to require several hundred million years to complete. It is believed that the Milky Way and Andromeda galaxies will eventually merge in similar fashion.

In applying logarithmic diffusion to this image, distinct generalized Linnik otfs $\hat{h}(\rho)=\left(1+\gamma \rho^{2 \sigma}\right)^{-\lambda}, \quad \gamma, \lambda>0,0<\sigma \leq 1$, were obtained for each RGB component. The $(\gamma, \lambda, \sigma)$ triplet for each component was as follows: red $=(0.195,0.692,0.85)$; green $=(0.201,0.643,0.88)$; and blue $=(0.151,0.684,0.83)$. We next solve the backwards in time logarithmic diffusion problem, $w_{t}=-\left[\lambda \log \left\{1+c(-\Delta)^{\sigma}\right\}\right] w, \quad c=$ $\gamma\left(4 \pi^{2}\right)^{-\sigma}, 0 \leq t \leq 1, w(x, y, 1)=g(x, y)$, using (22). In all three cases, with $K=100, s=0.001$, and $\bar{t}=0.75$, the $L^{1}$ norm was conserved on $1 \leq t \leq \bar{t}$, while the $T V$ norm increased by a factor of about 3.5 .

The Linnik image at the bottom of Figure 6 is noticeably brighter, and displays better resolution of the structural details in the two galaxies. Bluish young star clusters along spiral arms are better defined, together with dust lanes spiraling around the three cores of older stars. Background galaxies have become more visible. As a result, significant reduction in Lipschitz exponents was recorded. For the blue component, the original image has Lip $\alpha=0.28$, while the Linnik image has Lip $\alpha=0.15$. Lévy deblurring of the blue image resulted in $\operatorname{Lip} \alpha=0.18$.

3. Starburst galaxy NGC 3310. The example at the top of Figure 7 is a September 2001 Hubble telescope image of the Starburst galaxy NGC 3310, acquired using the Wide Field and Planetary Camera 2. That galaxy is of great interest to astronomers as it is known to produce clusters of new stars at a prodigious rate. Several hundred such clusters are visible as bright blue diffuse objects around the galaxy's spiral arms, each cluster representing up to a million stars.

In applying logarithmic diffusion to this image, the Linnik otf parameters for the red component were found to be $\gamma=0.328, \lambda=0.557$, and $\sigma=0.92$. The red and blue otfs almost coincided, while the green otf differed slightly. Accordingly, the red image otf was used for all three components. We next solve the backwards in time logarithmic diffusion problem, $w_{t}=-\left[\lambda \log \left\{1+c(-\Delta)^{\sigma}\right\}\right] w, \quad c=\gamma\left(4 \pi^{2}\right)^{-\sigma}, 0 \leq t \leq$ $1, w(x, y, 1)=g(x, y)$, using (22). In all three cases, with $K=40.0, s=0.001$, and $\bar{t}=0.75$, the $L^{1}$ norm increased by about $3 \%$ on $1 \leq t \leq \bar{t}$, with a threefold increase in $T V$ norm.

The Linnik deblurred image at the bottom of Figure 7 shows significant recovery of blue clusters of young stars at the periphery of the image; also, there is noticeable 
enhancement of structural detail in the main body of the galaxy, and in the surrounding dust lanes. In contrast, Lévy deblurring tends to produce saturation and loss of resolution near the core, as previously shown in Figure 4. For the red component, the original image has Lip $\alpha=0.26$, while the Linnik image has Lip $\alpha=0.14$. The Lévy image has Lip $\alpha=0.16$.

9. Scanning electron micrographs. There are other important classes of images which display the monotone convex behavior described in section 2 , and for which logarithmic diffusion deconvolution may be useful. One such class is scanning electron micrographs. In this section, we revisit experiments previously described in [11], and we verify that Linnik otfs can improve substantially on previous results. The two images considered here were taken by John Small at NIST. They are micrographs of a complex multiform crystalline compound of mercury. The author expresses his gratitude to Dr. David S. Bright (NIST) for making these images available.

The example at the top of Figure 8 has complex crystalline structure and fine scale surface detail that are of interest. The Linnik otf parameters for $\log |\hat{g}(\xi, 0)|$ were found to be $\gamma=0.0266, \lambda=0.5496$, and $\sigma=0.89$. We next solve the backwards in time logarithmic diffusion problem, $w_{t}=-\left[\lambda \log \left\{1+c(-\Delta)^{\sigma}\right\}\right] w, \quad c=\gamma\left(4 \pi^{2}\right)^{-\sigma}, 0 \leq$ $t \leq 1, \quad w(x, y, 1)=g(x, y)$, using (22). With $K=50.0, s=0.001$, and $\bar{t}=0.6$, the $L^{1}$ norm was conserved on $1 \leq t \leq \bar{t}$, with a threefold increase in $T V$ norm. The Linnik image at the bottom of Figure 8 shows recovery of numerous surface particles and other detail, and the complex morphology has become better defined. Deblurred image quality is particularly noticeable in this example. The original image has Lip $\alpha=0.37$, and the Linnik image has $\operatorname{Lip} \alpha=0.20$. The Lévy image was terminated at $\bar{t}=0.7$ and had Lip $\alpha=0.25$.

The slab like structure at the top of Figure 9 also exhibits interesting surface detail. The Linnik otf parameters were found to be $\gamma=0.022, \lambda=0.776$, and $\sigma=0.86$. With $K=50.0, s=0.001$, and $\bar{t}=0.7$, the $L^{1}$ norm was conserved on $1 \leq t \leq \bar{t}$, with a threefold increase in $T V$ norm. Here again, slab surface details are well-recovered, and the two corner structures reveal interesting small scale granularity. The original image has $\operatorname{Lip} \alpha=0.48$, and the Linnik image has $\alpha=0.25$. The Lévy image was terminated at $\bar{t}=0.75$ and had Lip $\alpha=0.34$.

Table 1 below summarizes the changes in image Lipschitz exponents recorded in each of the six deconvolution experiments in this paper. Linnik deblurring consistently produces smaller Lipschitz exponents than does Lévy deblurring. The last column in Table 1 is especially noteworthy.

\section{TABLE 1}

Lipschitz exponents before and after deconvolution

\begin{tabular}{|c|c|c|c|c|}
\hline Image & Original Lip & Lévy Lip & Linnik Lip & Linnik/Orig \\
\hline M 51 & $\alpha=0.39$ & $\alpha=0.21$ & $\alpha=0.13$ & $33 \%$ \\
\hline NGC 1309 & $\alpha=0.25$ & $\alpha=0.12$ & $\alpha=0.09$ & $36 \%$ \\
\hline NGC 6050 & $\alpha=0.28$ & $\alpha=0.18$ & $\alpha=0.15$ & $53 \%$ \\
\hline NGC 3310 & $\alpha=0.26$ & $\alpha=0.16$ & $\alpha=0.14$ & $54 \%$ \\
\hline SEM Crystal & $\alpha=0.37$ & $\alpha=0.25$ & $\alpha=0.20$ & $54 \%$ \\
\hline SEM Slab & $\alpha=0.48$ & $\alpha=0.34$ & $\alpha=0.25$ & $52 \%$ \\
\hline
\end{tabular}


10. Concluding remarks. A priori knowledge about the solution is an essential element in the successful computation of ill-posed inverse problems. Such knowledge informs the construction of the blind deconvolution procedure discussed in this paper. This methodology was shown to be capable of producing credible reconstructions in two distinct classes of real blurred images with real noise, one at the nanoscale, and the other at the cosmological scale. These two classes are acquired using distinct imaging modalities and are of considerable scientific interest. The accompanying sizeable reduction in Lipschitz exponents is highly significant.

This work substantially improves on previous work on the same classes of images [10], [11], based on isotropic Lévy stable otfs. Low Lévy exponents were found necessary to accomodate the Fourier behavior in the blurred image data. As shown in section 3, Lévy stable motions are related to Brownian motion through subordination. The present work is based on a new class of otfs that have the same high frequency behavior as low exponent stable otfs, but behave like high exponent stable laws near the origin. Such generalized Linnik otfs result from subordination of Lévy stable motions by the Gamma process, and their behavior near the origin was shown to play a vital role in fine structure recovery. The compelling quality of the new reconstructions strongly suggests that the detected generalized Linnik otfs that produce these results, must emulate essential aspects of the true system otfs in the two classes of images. Linnik otfs are not currently known in image analysis.

Sixty years after its introduction in [5], Bochner's seminal mathematical idea continues to yield a rich harvest of important applications.

\section{REFERENCES}

[1] C. Albanese and S. Lawi Time quantization and q-deformations, J. Phys. A: Math. Gen. 37 (2004), pp. 2983-2987.

[2] L. Bar, N. Sochen, And N. Kiryati, Semi-blind image restoration via Mumford-Shah regularization, IEEE Transactions on Image Processing, 15 (2006), pp. 483-493.

[3] P. Barthelemy, J. Bertolotti, and D. S. Wiersma, A Lévy flight for light, Nature 453 (2008), pp. 495-498.

[4] S. Bochner, Monotone Funktionen, Stieltjes Integrale und harmonische Analyse, Math. Ann., 108 (1933), pp. 378-410.

[5] S. Bochner, Diffusion equation and stochastic processes, Proc. Nat. Acad. Sci. USA 35 (1949), pp. $368-370$.

[6] S. Bochner, Harmonic Analysis and the Theory of Probability, University of California Press, Berkeley, CA 1955.

[7] A. S. CARAsso, The APEX method in image sharpening and the use of low exponent Lévy stable laws, SIAM J. Appl. Math. 63 (2002), pp. 593-618.

[8] A. S. Carasso, Singular integrals, image smoothness, and the recovery of texture in image deblurring, SIAM J. Appl. Math. 64 (2004), pp. 1749-1774.

[9] A. S. Carasso, False characteristic functions and other pathologies in variational blind deconvolution. A method of recovery, SIAM J. Appl. Math. 70 (2009), pp. 1097-1119.

[10] A. S. CARAsso, APEX blind deconvolution of color Hubble space telescope imagery and other astronomical data, Optical Engineering 45 (2006), 107004.

[11] A. S. Carasso, D. S. Bright, ANd A. E. Vladár, APEX method and real-time blind deconvolution of scanning electron microscope imagery, Optical Engineering 41 (2002), pp. 2499-2514.

[12] A. S. Carasso and T. Kato, On subordinated holomorphic semigroups, Trans. Amer. Math. Soc. 327 (1991), pp. 867-878.

[13] A. S. CARASSO AND A. E. VladÁR, Calibrating image roughness by estimating Lipschitz exponents, with appplications to image restoration, Optical Engineering 47 (2008), 037012.

[14] T. F. Chan And C. K. Wong, Total variation blind deconvolution, IEEE Transactions on Image Processing 7 (1998), pp. 370-375. 
[15] I. Eliazar And J. Klafter, Lévy, Ornstein-Uhlenbeck, and subordination: spectral vs jump description, Journal of Statistical Physics 119 (2005), pp. 165-196.

[16] M. B. Erdogan and I. V. OstrovskiI, Analytic and asymptotic properties of generalized Linnik probability densities, J. Math. Anal. Appl. 217 (1998), pp. 555-578.

[17] G. Fasshauer, Meshfree Approximation Methods with MATLAB, Interdisciplinary Mathematical Sciences - Vol. 6, World Scientific Publishers, Singapore, 2007.

[18] W. Feller, An Introduction to Probability Theory and its Applications, Vol. 2, Second ed., Wiley, New York, 1971.

[19] V. Ganti, A. Singh, P. Passalacqua, and E. Foufoula-Georgiou, Subordinated Brownian motion model for sediment transport, Physical Review E 80 (2009), 011111 pp 1-9.

[20] H. Geman, D. P. Madan, And M. Yor, Time changes for Lévy processes, Mathematical Finance 11 (2001), pp. 79-96.

[21] C. B. Johnson, Classification of electron-optical device modulation transfer function, Advances in Electronics and Electron Physics 33B (1972), pp. 579-584.

[22] C. B. Johnson, MTFs: a simplified approach, Electro-Optical Systems Design 4 (1972), pp. $22-26$.

[23] C. B. Johnson, Point-spread functions, line-spread functions, and edge-response functions associated with mtfs of the form $\exp \left[-\left(\omega / \omega_{c}\right)^{n}\right]$, Applied Optics, 12 (1973), pp. 10311033.

[24] L. Justen and R. Ramlau, A non-iterative regularization approach to blind deconvolution, Inverse Problems 22 (2006), pp. 771-800.

[25] J. Lee And D. SheEn, F. John's stability conditions versus A. Carasso's SECB constraint for backward parabolic problems, Inverse Problems 25 (2009), 055001.

[26] S. C. Lim And L. P. TeO, Analytic and asymptotic properties of multivariate generalized Linnik probability densities, arXiv:0903.5344v1 [math.PR] 30 Mar 2009.

[27] E. Lukacs, Characteristic Functions, Second ed., Griffin, London, 1970.

[28] D. P. Madan and E. Seneta, The Variance Gamma model for share market returns, Journal of Business 63 (1990), pp. 511-524.

[29] D. P. Madan, P. P. CARR, And E. C. Chang, The Variance Gamma process and option pricing, European Finance Review 2 (1998), pp. 79-105.

[30] R. S. PhILLIPS, On the generation of semigroups of linear operators, Pacific J. Math. 2 (1952), pp. 343-369.

[31] G. Samorodnitsky and M. S. Taqqu, Stable Non-Gaussian Random Processes: Stochastic Models with Infinite Variance, Chapman and Hall, New York, 1994.

[32] K. Sato, Lévy Processes and Infinitely Divisible Distributions, Cambridge University Press, 1999.

[33] I. J. Schoenberg, Metric spaces and completely monotone functions, Ann. of Math. 39 (1938), pp. 811-841.

[34] M. H. TAIBlESON, On the theory of Lipschitz spaces of distributions on Euclidean n-space. I. Principal properties, Journal of Mathematics and Mechanics 13 (1964), pp. 407-478.

[35] A. Weron and M. Magdziarz, Anomalous diffusion and semimartingales, Europhysics Letters 86 (2009), 60010 pp. 1-6. 